\title{
Approximate Aerodynamic and Aeroelastic Modeling of Flapping Wings in Hover and Forward Flight
}

\author{
Abhijit Gogulapati * and Peretz P. Friedmann ${ }^{\dagger}$ \\ Department of Aerospace Engineering, The University of Michigan, Ann Arbor, 48109, USA
}

\begin{abstract}
Results generated from an aeroelastic model that combines a nonlinear structural dynamic model based on MARC with an approximate aerodynamic model that incorporates leading edge vortices and a wake model are presented. The aerodynamic model, used in our earlier studies, is extended to forward flight, and the effect of fluid viscosity is incorporated into the formulation in a partial manner. Results presented describe aerodynamic and aeroelastic studies conducted on airfoils and flapping wings in hover and forward flight. For the rigid cases considered, the approximate model shows reasonable agreement with CFD based results and predicts the trends accurately. The aeroelastic results presented indicate that wing flexibility has beneficial effects in both hover and forward flight. Comparisons show that aerodynamic loads are comparable to inertia loads for the cases considered.
\end{abstract}

\section{Nomenclature}

\begin{tabular}{ll}
$A_{1}-A_{11}$ & Coefficients that are computed from the airfoil degrees of freedom \\
$A_{f}, B_{f}$ & Filter coefficients \\
$A_{w}$ & Wing area \\
$\boldsymbol{d} \boldsymbol{A}_{s p}$ & Instantaneous area vector in the $\left(X_{S P}, Y_{S P}, Z_{S P}\right)$ coordinate system \\
$a$ & Distance between pitching axis and mid-chord of the airfoil \\
$C_{D}$ & Drag coefficient \\
$C_{L}$ & Lift coefficient \\
$C_{T}$ & Thrust coefficient \\
$c$ & airfoil chord \\
$c_{r}$ & Root chord \\
$D$ & Drag \\
$E$ & Elastic modulus \\
$\boldsymbol{e}$ & Unit vector; subscript identifies the direction. \\
$\boldsymbol{F}_{s p}$ & Force vector in the $\left(X_{S P}, Y_{S P}, Z_{S P}\right)$ coordinate system \\
$F_{h}, F_{v}$ & Components of the aerodynamic forces in the horizontal and vertical directions \\
$f$ & Flapping frequency \\
$h$ & Plunge degree of freedom of the airfoil \\
$j, k$ & Indices \\
$L$ & Lift \\
$L_{C}$ & Circumference of the airfoil \\
$l$ & Lead-lag degree of freedom of the airfoil \\
$l_{0}$ & Lead-lag amplitude \\
$M$ & User defined number of sections \\
$m_{f}$ & Order of the filter \\
$N_{\text {sections }}$ & Total number of aerodynamic span stations on the wing \\
$N_{\theta}$ & Discretization of the circle \\
$n_{w k s u b i t}$ & Number of wake sub-iterations \\
$p$ & Local static pressure on the airfoil \\
\hline
\end{tabular}

${ }^{*}$ Ph.D. candidate, Student Member AIAA.

${ }^{\dagger}$ François-Xavier Bagnoud Professor, Fellow AIAA. 


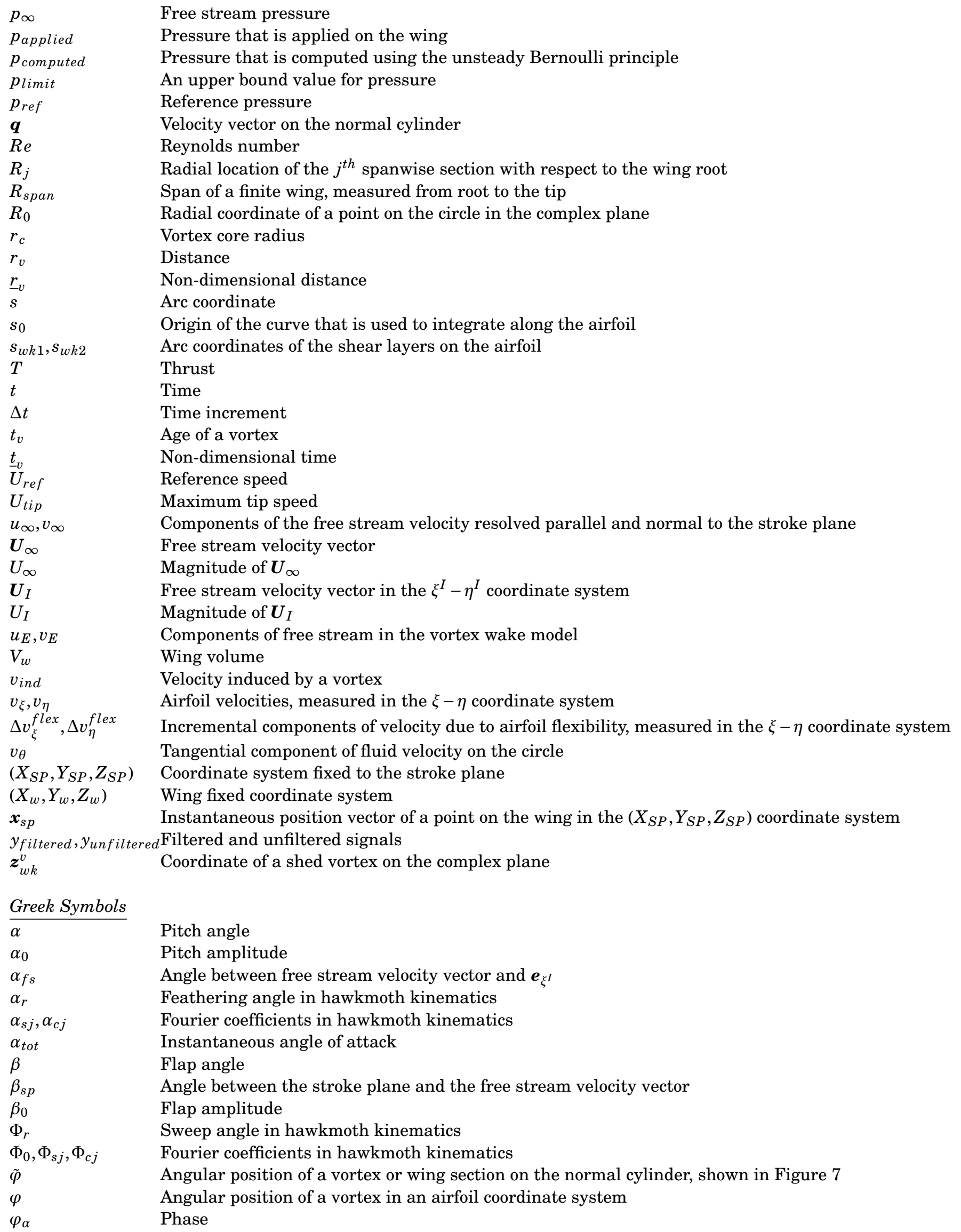




\begin{tabular}{|c|c|}
\hline$\phi$ & Velocity potential \\
\hline$l$ & $\sqrt{-1}$ \\
\hline$\psi$ & Rotation vector \\
\hline$\zeta$ & Coordinate of a point in an airfoil coordinate system on the normal cylinder \\
\hline$\Gamma_{f s}$ & Circulation due to free stream \\
\hline$\Gamma_{0}$ & Quasi-steady circulation \\
\hline$\gamma_{b}$ & Bound vorticity \\
\hline$\gamma_{f s}$ & Component of vorticity due to free stream \\
\hline$\gamma_{u s}$ & Component of vorticity due to airfoil velocities \\
\hline$\gamma_{w i}$ & Component of vorticity on the circle that is induced by the wake \\
\hline $\mathrm{d} \Gamma_{w k}^{v}$ & Circulation a vortex \\
\hline$\Theta_{r}$ & Elevation angle in hawkmoth kinematics \\
\hline$\Theta_{0}, \Theta_{s j}, \Theta_{c j}$ & Fourier coefficients in hawkmoth kinematics \\
\hline$\theta$ & Angular coordinate on the circle in the complex plane \\
\hline$\tau, \sigma$ & Thickness and camber parameters in the aerodynamic formulation \\
\hline$\mu$ & Advance ratio \\
\hline$v_{\infty}$ & Kinematic viscosity of the fluid \\
\hline$v$ & Poisson's ratio \\
\hline$\omega$ & Circular frequency \\
\hline$\rho$ & Density of the material \\
\hline$\rho_{\infty}$ & Free stream density of the fluid \\
\hline$(\xi, \eta)$ & Coordinates in an airfoil coordinate system; superscript identifies the coordinate system \\
\hline$\varsigma$ & Dummy variable, used in integration \\
\hline \multicolumn{2}{|c|}{ Additional Subscripts and Superscripts } \\
\hline$|\cdot|$ & Absolute value or magnitude \\
\hline$(\ddot{\bullet})$ & Double derivative with respect to time \\
\hline$\left.(\cdot)\right|_{\text {inviscid }}$ & Inviscid quantity \\
\hline$\left.(\cdot)\right|_{\text {viscous }}$ & Viscous quantity \\
\hline$(\cdot)^{*}$ & Complex conjugate \\
\hline$(\cdot)^{\text {flex }}$ & Quantity corresponding to a flexible airfoil \\
\hline
\end{tabular}

\section{Introduction}

During the last fifteen years there has been increasing interest in micro air vehicles (MAVs) for both military and civilian missions that involve confined spaces, such as buildings, or short distances. These vehicles typically have maximum geometric dimensions of $15 \mathrm{~cm}$, maximum weight of 100 grams, and are expected to operate at low Reynolds number $\left(10^{2}<\operatorname{Re}<10^{4}\right)$ and low forward flight speed $(<15 \mathrm{~m} / \mathrm{s}) .{ }^{1}$ In particular, flapping wing designs, inspired by hover-capable biological flyers such as insects, bats, and hummingbirds, are particularly interesting due to the exceptional flight capabilities observed in the biological counterparts. ${ }^{1}$

A significant portion of the research on flapping wing vehicles has focused on understanding the mechanisms that generate unsteady aerodynamic forces. This research ${ }^{1-7}$ has identified leading edge vortices (LEVs), wake capture, and tip vortices, as the primary force generating mechanisms. Recent emphasis has been on investigating the dynamics of LEVs, and its interaction with kinematics. ${ }^{8}$ Water tunnel experiments, conducted using airfoils undergoing combined pitch-plunge motion, showed that the behavior of LEVs was dependent on the reduced frequency for a given effective angle of attack. ${ }^{8}$ In these tests, flow field measurements were done using particle induced velocimetry (PIV), and the effective angle of attack was defined based on the combined pitch-plunge motion. Attempts to model the aerodynamic environment in a quantitative manner have been based on two approaches: (1) computational fluid dynamics (CFD) simulations based on the solution of the Navier Stokes (NS) equations and (2) approximate aerodynamic models based on potential flow solutions. Simulations using CFD yield the best resolution of the unsteady flow field. However, such approaches require significant amounts of computer time that prevent parametric studies. Approximate aerodynamic models offer a compromise between accuracy and computational efficiency and thus are suitable for trend and design studies. It is important to emphasize that the approximate models that have practical 
applications have to be able to model the effect of LEVs and wake capture.

The approximate unsteady aerodynamic theories used for flapping wing problems can be classified as assumed (or prescribed) wake and free wake models. Assumed wake models are classical unsteady models such as Theodorsen's theory. ${ }^{9}$ Reference [10] incorporated the effect of the LEVs in Theodorsen's theory by modifying the unsteady aerodynamic lift and moment expressions using the Polhamus leading edge suction analogy. ${ }^{11}$ This mode ${ }^{10}$ was compared to experiments and was capable of predicting the trends in aerodynamic forces.

Free wake models account for evolution of the wake, thereby providing a reasonable approximation to the development of the unsteady wake during a flapping cycle. In particular, free wake models that account for LEVs are two-dimensional formulations that use a discrete vortex representation of the wake. ${ }^{12-14}$ These formulations are suitable for flapping wings in hover following simplifying assumptions on the geometry of the shed wake. The model developed in Ref. [12], which accounts for separation close to the leading edge, compared well with experimental data for airfoils in steady flow. In this approach the chordwise location of the separation point, which may be obtained using independent computations or experiments, is explicitly incorporated into the formulation. Reference [13] described a vortex blob based formulation that simulates the unsteady flow field around flexible thin airfoil that is undergoing prescribed rigid body motion as well as prescribed deformation. The model displayed good correlation with previously published data for rigid airfoils; however, the study indicated that additional work was required before the formulation could be used for aeroelastic studies. References $[14,15]$ presented the development and implementation of a discrete vortex model that is applicable to insect-like flapping wings in hover. The model was used to simulate rigid wings undergoing hover kinematics, and for the limited number of cases considered, compared well with experimental data. ${ }^{15}$

For the case of forward flight, the presence of a free stream alters the shed wake geometry and wing-wake interaction. Therefore, additional modifications are needed before two dimensional aerodynamic formulations $^{12-14}$ can be used to model forward flight. A review of literature indicates that the application of free wake models that incorporate LEVs to the problem of flapping wings in forward flight has received limited attention. Recently, Ref. [16] extended the aerodynamic formulation developed in Refs. [14,15] to forward flight using the concept of prescribed wake adopted from rotary wing aerodynamics. Preliminary results indicated that forward flight increased the peak force during one half of the stroke and reduced the force during the other half of the stroke; however, comparisons with CFD based or experimental results were not presented.

The importance of wing flexibility in enhancing the lift producing capability of flapping wings has been considered in a number of studies. Studies that have attempted to examine this issue in a systematic manner include Refs. [10,16-25]. An important finding of Refs. [17,18], which was later corroborated by experiments conducted in Ref. [10], was that a dominant component of the loading on flapping wings is due to inertia loads. Reference [10] examined wing models based on membranes reinforced by metal beams, using a linear structural model. It concluded that wing flexibility changed the aerodynamic loads and therefore should be included in the model. Reference [19] examined the influence of flexibility in insect wings by using a linear finite element model of a dragonfly wing that was combined with CFD based aerodynamic loading and found that spanwise flexibility had a favorable impact on thrust generated and power consumed by the flapping wing. Reference [16,20,21] described a nonlinear aeroelastic model that is obtained by coupling a nonlinear finite element model of the wing with an approximate model. These studies, which examined MAV wings undergoing prescribed motion found that the effect of aerodynamic loads was small compared to effect of inertia loads for the cases considered; wing flexibility had a small but favorable impact on lift generation. References [22,23] describe the development of a computational aeroelastic framework obtained by combining geometrically nonlinear beam and shell based structural dynamic models with a CFD based flow field. The framework was used in Ref. [24] to simulate isotropic Zimmerman wings in hover. The results indicated that the behavior of peak lift was non-monotonic with decrease in elastic modulus, and concluded that only a limited amount of flexibility was beneficial for thrust generation. Plunging isotropic plunging wings, offset by a constant flap rotation, in hover for $R e=100$ were studied in Ref. [25]. The aeroelastic model was obtained by coupling a viscous incompressible fluid description based on a lattice Boltzmann model to a structural description based on a lattice spring model. This study ${ }^{25}$ in which flexibility was incorporated using linear springs, found that a peak in lift coefficient coincided with an excitation frequency that was close to the resonant frequency of the wings. Combinations of elastic modulus and flapping frequency were found for which the lift generated was sufficient to support the weight of a realistic insect.

The overall objective of the current paper is to develop an understanding of the effect of flexibility on the performance of anisotropic flapping wings in hover and forward flight using an aeroelastic model, initially 
described in Refs. [16, 20,21], which has been extended to forward flight. The specific objectives of the paper are:

1. Extend the aerodynamic formulation to incorporate forward flight.

2. Include the effect of Reynolds number in a partial manner by incorporating the effect of temporal decay of vorticity once it is shed into the wake.

3. Compare results obtained using the modified aerodynamic model with those obtained using CFD for airfoils and wings in hover and forward flight.

4. Conduct trend type studies for flexible wings in hover and forward flight.

\section{Nonlinear Aeroelastic Model}

The aeroelastic model is obtained by coupling a nonlinear finite element model of the wing with an approximate aerodynamic model that incorporates LEVs and a free wake; a detailed description of the individual components and formulation of the aeroelastic equations of motion for flapping wings in hover was presented in Refs [20,21]. In this section, a summary of the aeroelastic model including modifications to the aerodynamic formulation to incorporate flapping wings in forward flight are presented.

\section{A. Structural Dynamic Model and Wing Kinematics}

The structural dynamic models for MAV wings are based on the MARC code ${ }^{26}$ using shell elements that are capable of undergoing large amplitude rigid body motion as well as moderate-to-large flexible deformation. A variety of constitutive laws are available so that isotropic as well as anisotropic wings may be modeled. Wing kinematics, which consist of large amplitude rigid body rotations prescribed at the root, are applied as displacement boundary conditions at one or more nodes.

\section{B. Approximate Aerodynamic Model}

The unsteady aerodynamic model that was selected for use and modification was developed in Refs. $[14,15]$. This formulation, originally derived for the case of hover, is based on two-dimensional potential flow and uses a vorticity/circulation approach to compute the aerodynamic loads. Several modifications were introduced by us. First, the model was modified to account for spanwise and chordwise flexibility of flapping wings as described in Refs. [20,21]. In the current paper, the aerodynamic formulation is extended to incorporate forward flight. Moreover, the effect of Reynolds number is also incorporated into the calculation of shed vorticity. Details of these extensions are provided next.

\section{Overview of the Aerodynamic Formulation}

The overall approach, which retains the essential aspects of the hover formulation for rigid and flexible wings, ${ }^{14,20,21}$ is summarized in Figure 1. First, the wing is divided into several spanwise stations, as shown in Figure 2, where each section is represented as an airfoil. For each airfoil, an airfoil-wake surface that captures the airfoil degrees of freedom (DOF), and approximates the geometry of the shed wake, is identified. Next, the airfoil and the airfoil-wake surface are transformed to a circle in the complex plane using a conformal mapping. Thus, the airfoil bound and shed wake vorticity are computed on the complex plane. The quasi-steady component of vorticity is obtained by neglecting the effect of the shed wake. The strength of shed vorticity is computed by enforcing a stagnation condition at the leading edge (LE) and a Kutta condition at the trailing edge (TE). The airfoil bound vorticity is obtained as a sum of the quasi-steady and wake-induced vorticity on the airfoil. Next, the vorticity in the complex plane is transformed back to the airfoil-wake surface (physical plane) using an inverse transform. The unsteady loads acting on the airfoil are obtained from the total vorticity using the unsteady Bernoulli equation. Finally, the shed vorticity is convected using the Rott-Birkhoff equation, which is derived from Biot-Savart law for two dimensional flow. 


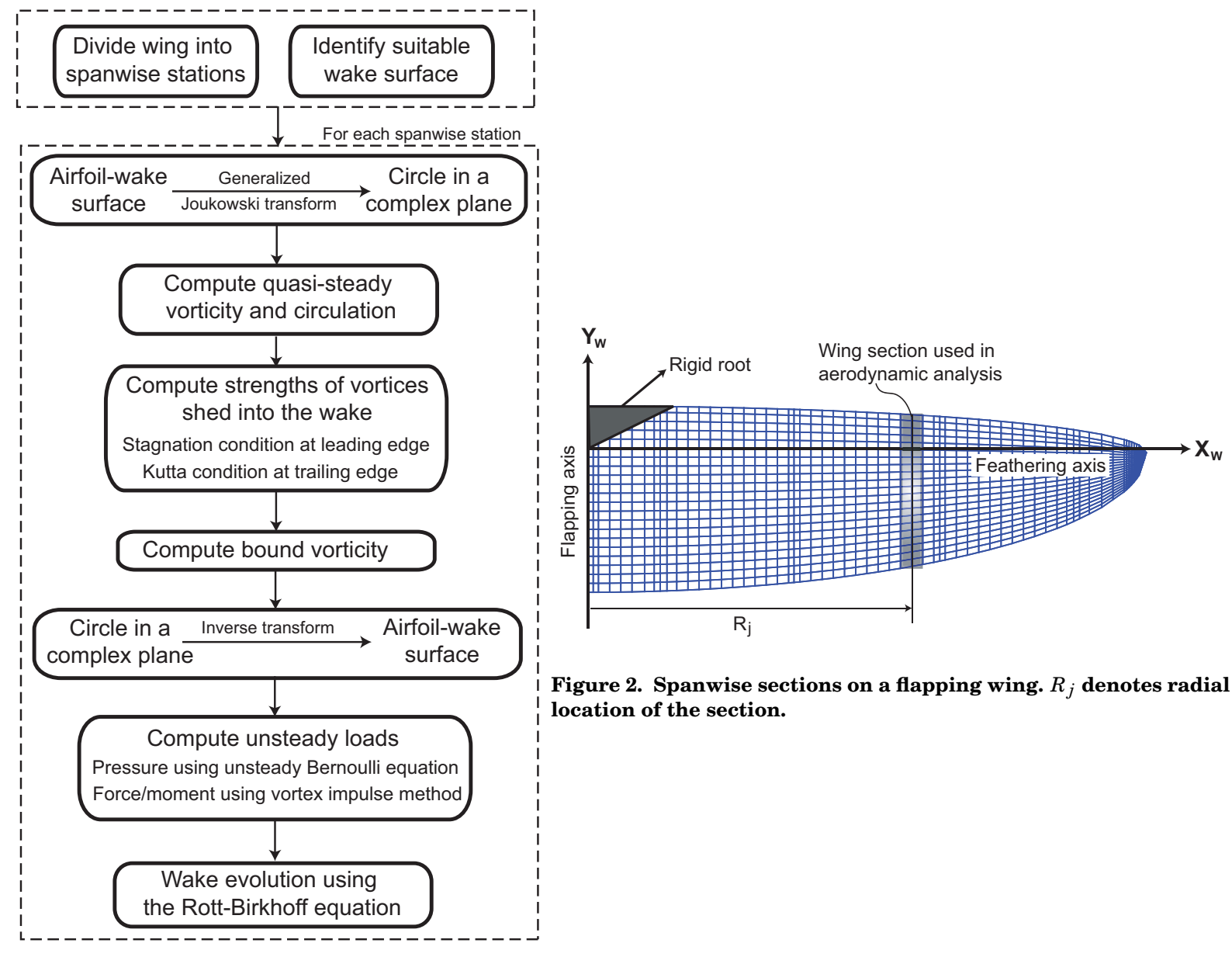

Figure 1. Schematic of the aerodynamic formulation.

\section{The Airfoil-Wake Surface}

Wing kinematics of biological flapping wing flyers, in both hover and forward flight, consists of a predominant sweep or flap motion in the stroke plane (SP), pitching about the feathering axis, and a comparatively small elevation angle, as indicated in Figure $3 .^{27}$ Therefore, the feathering axis of the wing is assumed to move on the stroke plane (SP). ${ }^{14}$ Consequently, the surface described by the airfoil motion is a cylinder that is normal to the stroke plane; this normal cylinder (NC) is shown in Figures 4 and 5.

For hover, ${ }^{14}$ the shed wake is assumed to be confined to the NC; therefore, $\mathrm{NC}$ is a convenient choice for the airfoil-wake surface. For the case of forward flight, the vortices shed into the wake are carried away with the free stream due to velocity of forward flight and therefore a suitable approximation to the wake surface has to be identified.

\section{Extension to Forward Flight}

Forward flight is characterized by a free stream velocity vector that is assumed to lie in the $Y_{S P}-Z_{S P}$ plane as depicted in Figure 4. A complete description of the free stream velocity involves two independent parameters $u_{\infty}$ and $v_{\infty}$. These quantities are determined from the tip speed, advance ratio, and stroke plane inclination angle, using Eq. (1).

$$
\mu=\frac{-u_{\infty}}{U_{t i p}} \quad \text { so that } \quad u_{\infty}=-\mu U_{t i p} \text { and } \quad v_{\infty}=u_{\infty} \tan \left(\beta_{s p}\right)
$$

where $u_{\infty}, v_{\infty}$, and $\beta_{s p}$ are shown in Figure 4 .

In the present analysis, the $\mathrm{NC}$, which is used for hover, is also used to approximate the geometry of the shed wake in forward flight. The effect of free stream due to forward flight is incorporated by modifying the 


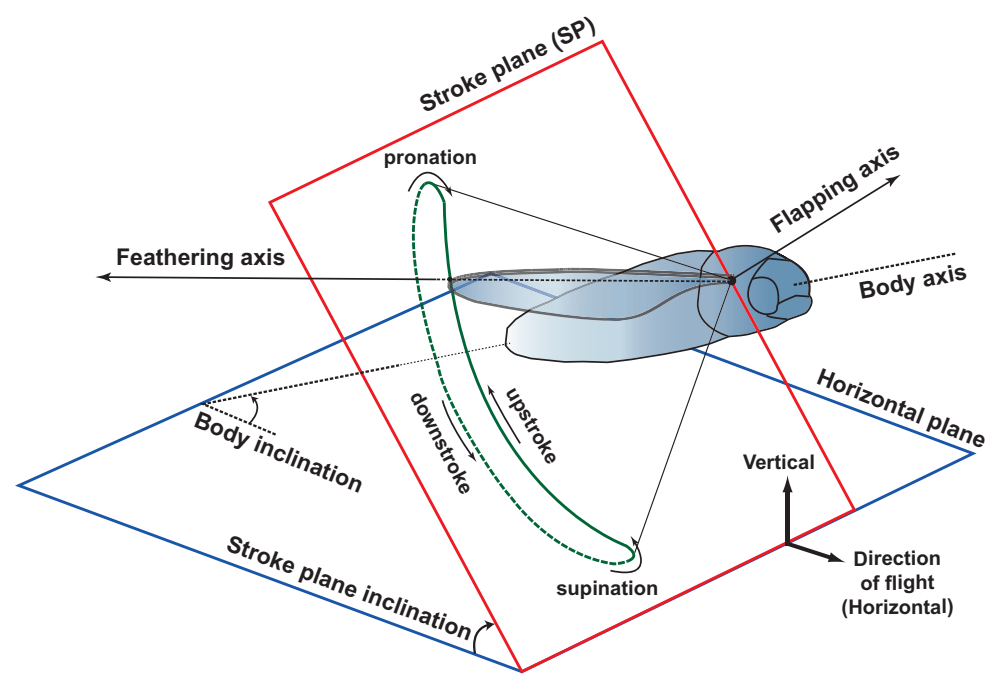

Figure 3. Insect flapping stroke

expressions used to determine the quasi-steady component of vorticity and the vortex wake model as described later.

\section{Coordinate Systems}

Several rectangular coordinate systems, which are defined on the flattened NC, are used for each airfoil section. These coordinate systems, shown in Figure 6, are listed below, where the unit vectors corresponding to the axes are denoted using $\boldsymbol{e}$ with an appropriate subscript:

1. $(\xi, \eta)$ : Zero lift coordinate system. Origin at the mid-chord; $\boldsymbol{e}_{\xi^{A}}$ along the zero lift line (ZLL); $\boldsymbol{e}_{\eta^{A}}$ normal to ZLL.

2. $\left(\xi^{I}, \eta^{I}\right)$ : Stationary coordinate system fixed to the NC; also shown in Figure 5. $\boldsymbol{e}_{\xi I}$ is parallel to the stroke plane; $\boldsymbol{e}_{\eta}$ is parallel to $Z_{S P}$. Identified by superscript $I$.

3. $\left(\xi^{n r}, \eta^{n r}\right)$ : Non-rotating coordinate system that translates with the airfoil. Origin at the intersection of the feathering axis of the wing and the normal cylinder. $\boldsymbol{e}_{\xi^{n r}}$ and $\boldsymbol{e}_{\eta^{n r}}$ are parallel to the stroke plane and $\boldsymbol{e}_{\eta^{I}}$ respectively. Identified by superscript $n r$.

4. $\left(\xi^{f a}, \eta^{f a}\right)$ : Coordinate system that is fixed to the center of rotation of the airfoil. Origin at the intersection of the feathering axis of the wing and the normal cylinder. $\boldsymbol{e}_{\xi f a}$ and $\boldsymbol{e}_{\eta^{f a}}$ are parallel to $\boldsymbol{e}_{\xi}$ and $\boldsymbol{e}_{\eta}$ respectively. Identified by superscript $f a$.

Each airfoil has three degrees of freedom (DOF) that are defined in $\xi^{I}-\eta^{I}$ on the flattened NC as shown in Figure 6; these are lead-lag $(l)$, plunge $(h)$, and pitch $(\alpha)$ respectively. The airfoil DOFs and velocities are obtained from the structural dynamic model at each time step; consequently, these quantities include the effect of wing deformation in addition to the effect of the wing kinematics. The effect of spanwise deformation on the radius of the NC is incorporated by using an average time dependent radius as follows: Let $R_{j}^{0}$ and $R_{j}^{t}$ denote the radial locations of a wing section at the start of the motion and at some subsequent time $t$. Then, the average radius of the normal cylinder at time $t$ is given by $\bar{R}_{j}^{t}=0.5\left(R_{j}^{0}+R_{j}^{t}\right)$. Subsequently, $\bar{R}_{j}^{t}$ is used to calculate the distances on the NC.

\section{Quasi-steady Vorticity}

Quasi-steady vorticity, ${ }^{14}$ computed on the circle, is obtained as a sum of two components: (a) a free stream component that is computed from the instantaneous angle of attack and free stream velocity, and (b) an unsteady component that is computed from airfoil velocities. 


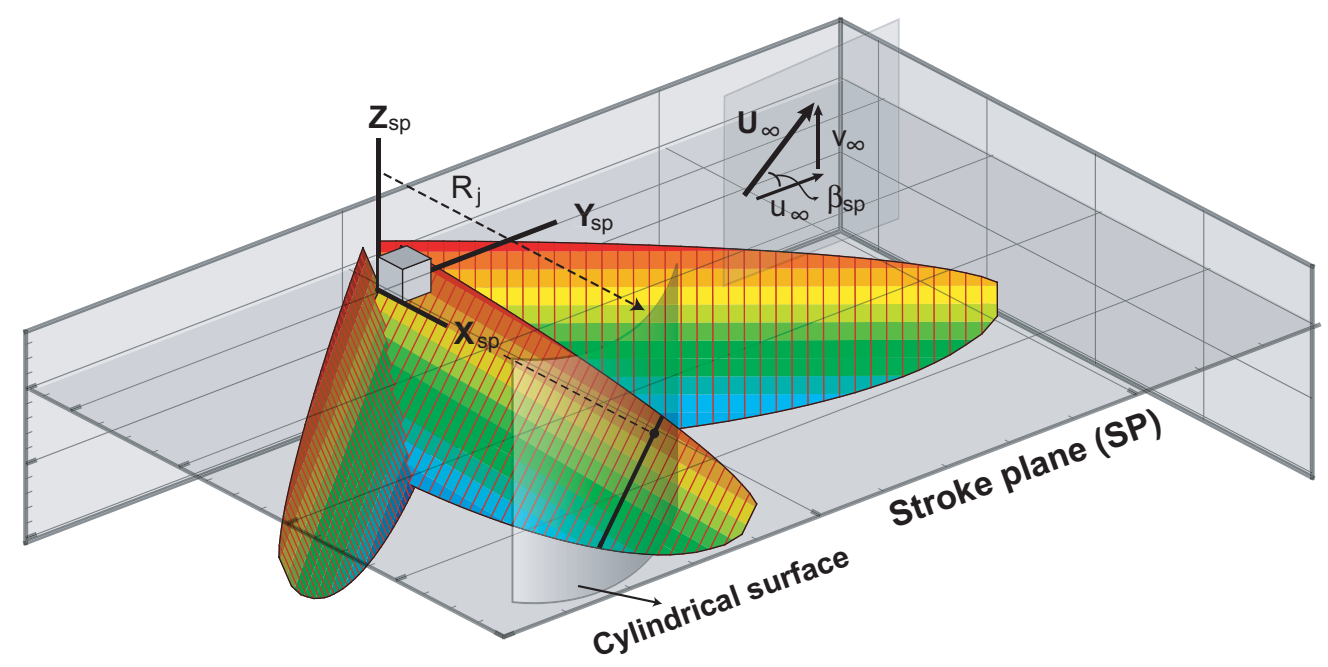

Figure 4. Coordinate system that is fixed to the stroke plane. Free stream velocity vector and cylindrical surface are also shown.

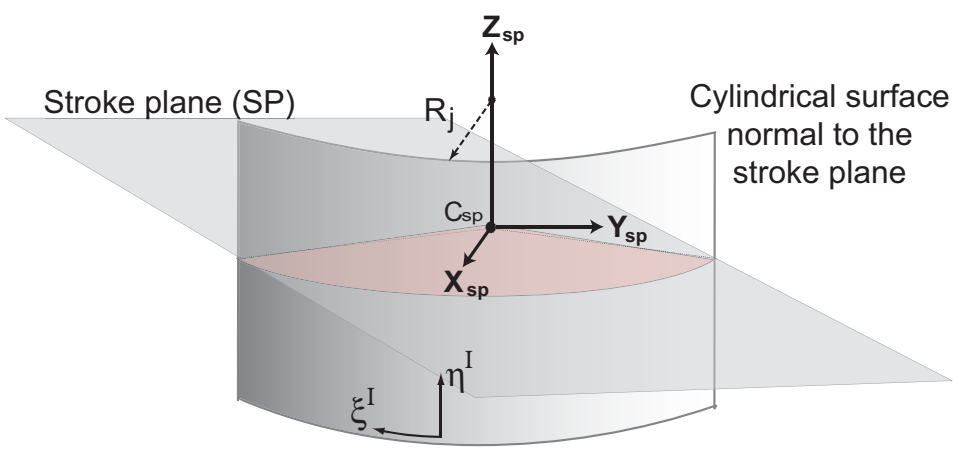

Figure 5. Normal cylinder described by the wing section.

Free stream component of vorticity

The vorticity and circulation due to a free stream, derived in Ref. [14], are

$$
\begin{aligned}
\gamma_{f s}(\theta, t) & =-2 u_{I}[\sin (\theta-\alpha)+\sin (\alpha)] \\
\Gamma_{f s}(t) & =-4 \pi R_{0} u_{I} \sin (\alpha)
\end{aligned}
$$

where the vorticity and circulation are positive in the counter-clockwise direction as indicated in Figure 6 . In Ref. [14], the analysis was limited to hover; therefore, it was assumed that $u_{I} \equiv 0$ and Eq. (2) did not contribute to the quasi-steady component of vorticity. In the current study, the effect of free stream velocity is incorporated into Eq. (2) as follows.

The free stream velocity vector, and its magnitude, at each wing section are given by

$$
\begin{aligned}
\boldsymbol{U}_{I} & =-u_{\infty} \cos \tilde{\varphi}_{w} \boldsymbol{e}_{\xi I}+v_{\infty} \boldsymbol{e}_{\eta^{I}} \\
U_{I} & =\left\|\boldsymbol{U}_{I}\right\|=\sqrt{\left(u_{\infty} \cos \tilde{\varphi}_{w}\right)^{2}+v_{\infty}^{2}}
\end{aligned}
$$

where $\tilde{\varphi}$ is depicted in Figure 7 and subscript $w$ indicates wing section. The instantaneous angle of attack of the airfoil, which is equal to the angle between the free stream velocity vector and the $\xi$ axis as shown in Figure 6, is:

$$
\alpha_{t o t}=\alpha+\alpha_{f s} \quad \text { where } \quad \alpha_{f s}=\tan ^{-1}\left(\frac{v_{I}}{u_{I}}\right)=\tan ^{-1}\left(-\frac{v_{\infty}}{u_{\infty} \cos \tilde{\varphi}_{w}}\right)
$$


Therefore, the modified expressions for vorticity and circulation due to the free stream velocity and angle of attack are given by

$$
\begin{aligned}
\gamma_{f s}(\theta, t) & =-2 U_{I}\left[\sin \left(\theta-\alpha-\alpha_{f s}\right)+\sin \left(\alpha+\alpha_{f s}\right)\right] \\
\Gamma_{f s}(t) & =-4 \pi R_{0} U_{I} \sin \left(\alpha+\alpha_{f s}\right)
\end{aligned}
$$

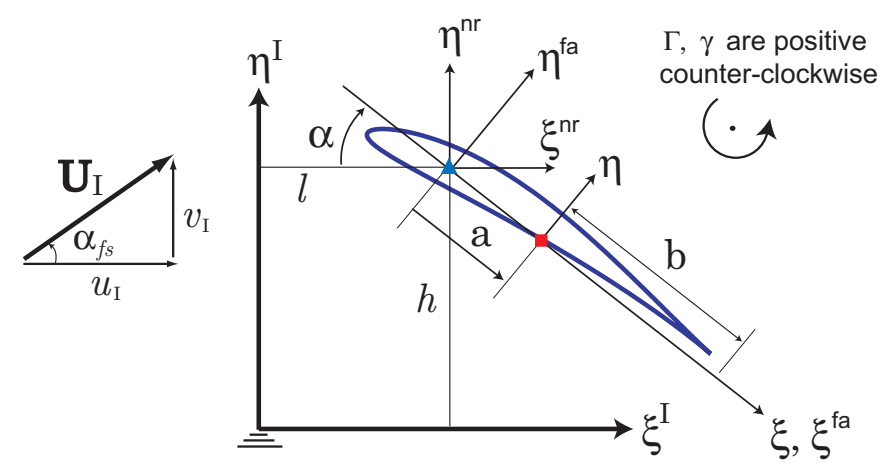

Figure 6. Degrees of freedom of the airfoil and coordinate systems used.

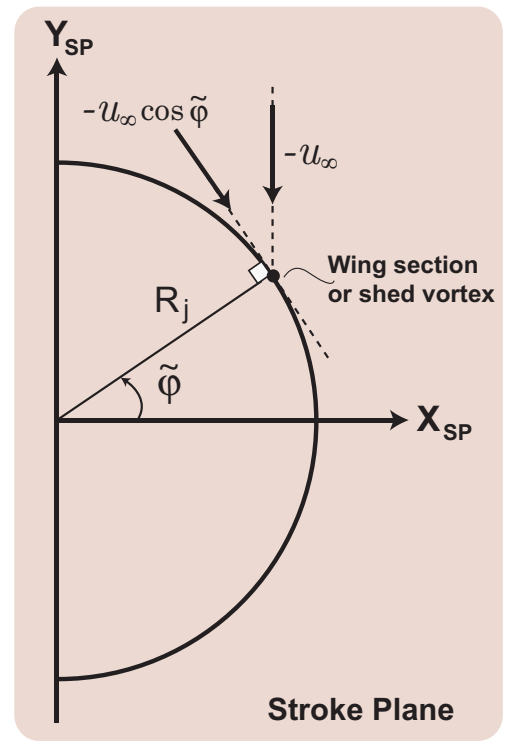

Figure 7. Component of $u_{\infty}$ normal to the instantaneous position of the wing. .

\section{Unsteady component of vorticity}

The derivation of the unsteady component is identical to the one presented in Ref. [21]. The expression for the tangential velocity of the fluid on the surface of a flexible airfoil is given by

$$
\begin{aligned}
\left.v_{\theta}(\theta, t)\right|_{u s} ^{\text {flex }}= & \frac{1}{R_{0}}\left[-A_{1} \cos \theta-\left(A_{2}+\frac{1}{2} A_{7}\right) \cos 2 \theta+A_{3} \sin \theta+\left(A_{4}-\frac{1}{2} A_{5}+\frac{1}{2} A_{6}\right) \sin 2 \theta\right. \\
& \left.-A_{8} \sin \theta \cos 2 \theta+A_{9} \sin \theta \sin 2 \theta-A_{10} \cos \theta \cos 2 \theta+A_{11} \cos \theta \sin 2 \theta\right] \\
& +\frac{\Delta v_{\xi}^{\text {flex }}}{R_{0}}[\tau(\sin \theta-\sin 2 \theta)+\sigma(\cos \theta-\cos 2 \theta)]+ \\
& +\frac{\Delta v_{\eta}^{\text {flex }}}{R_{0}}\left[-2 R_{0} \cos \theta+\tau(\cos \theta-\cos 2 \theta)-\sigma(\sin \theta-\sin 2 \theta)\right]
\end{aligned}
$$

and the vorticity on the circle is obtained as

$$
\gamma_{u s}^{\text {flex }}(\theta, t)=\left.v_{\theta}(\theta, t)\right|_{u s} ^{f l e x}+\frac{\Gamma_{0}}{2 \pi R_{0}}
$$

where

$$
\Gamma_{0}=2 \pi\left[2 R_{0}(i \sin \alpha+\dot{h} \cos \alpha)+\dot{\alpha}\left(\frac{1}{2} \tau^{2}+\frac{1}{2} \sigma^{2}-2 R_{0}\left(R_{0}+a\right)\right)\right]
$$

\section{Effect of Fluid Viscosity in Shed Vorticity}

In a viscous fluid, the influence of shed vorticity at a point of interest decays with increase in distance as well as the age of the vortex. At sufficiently large Reynolds numbers, the temporal decay of the vortex strength 
is slow enough compared to the time of simulation so that one may assume that the strength of the vortex is constant with respect of time. However, this effect needs to re-examined at lower Reynolds number.

In this study, the decay of vortex strength is incorporated in the expression of induced velocity due to shed vorticity. From Ch. 13, Ref. [28], the induced velocity due to a viscous shed vortex is given by the following expression:

$$
\left.v_{\text {ind }}\right|_{\text {viscous }}=\left.v_{\text {ind }}\right|_{\text {inviscid }}\left(1-e^{-\frac{r_{v}^{2}}{4 v_{\infty} t_{v}}}\right)=\left.v_{\text {ind }}\right|_{\text {inviscid }}\left(1-e^{-R e \frac{r_{v}^{2}}{4 t_{v}}}\right)
$$

where $r_{v}$ denotes the distance between the vortex and the point at which induced velocity is computed, $t_{v}$ denotes the age of the vortex, and

$$
R e=\frac{U_{r e f} c}{v_{\infty}} \quad ; \quad \underline{r}_{v}=\frac{r_{v}}{c} \quad ; \quad \underline{t}_{v}=\frac{t_{v} U_{r e f}}{c}
$$

Incorporating this effect into the aerodynamic model modifies the constraint conditions used to determine the shed vorticity, the expressions used to computed wake induced and bound vorticity, the evolution of the wake, and the calculation of the pressure using the unsteady Bernoulli equation; ingredients of the model are discussed next.

\section{Determination of Shed Vorticity}

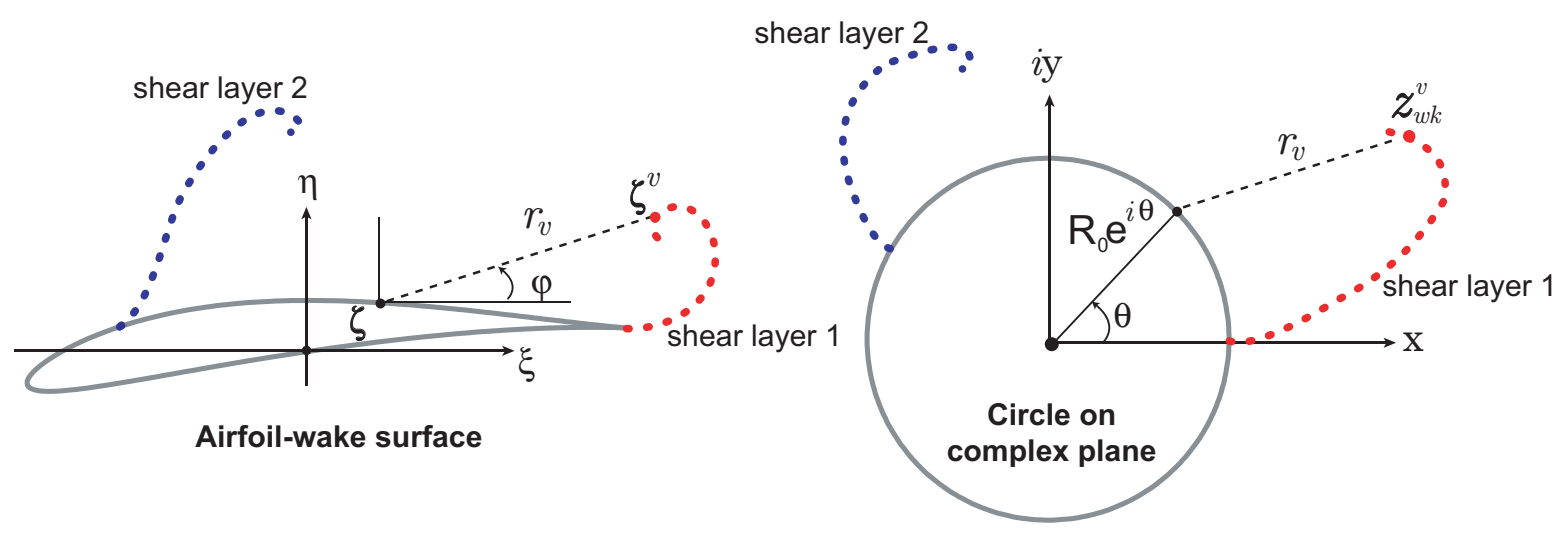

Figure 8. Distances and angles that are used in the computation of velocity potential.

Following Ref. [14], the induced velocity on the circle due to an inviscid 2D vortex shed into the wake is given as follows

$$
\left.v_{\text {ind }}(\theta, t)\right|_{\text {inviscid }}=-\frac{1}{2 \pi R_{0}} \oint_{w k} \mathscr{R}\left(\frac{\boldsymbol{z}_{w k}^{v}+R_{0} \mathrm{e}^{\imath \theta}}{\boldsymbol{z}_{w k}^{v}-R_{0} \mathrm{e}^{\imath \theta}}\right) \mathrm{d} \Gamma_{w k}^{v}
$$

where, $\mathscr{R}$ denotes real part, $w k=w k 1+w k 2$, and $w k 1$ and $w k 2$ denote shear layers emanating from the airfoil shown in Figure 8. Combining Eqs. (7) and Eq. (8), the induced velocity becomes

$$
\left.v_{\text {ind }}(\theta, t)\right|_{\text {viscous }}=-\frac{1}{2 \pi R_{0}} \oint_{w k}\left[\mathscr{R}\left(\frac{\boldsymbol{z}_{w k}^{v}+R_{0} \mathrm{e}^{\imath \theta}}{\boldsymbol{z}_{w k}^{v}-R_{0} \mathrm{e}^{\imath \theta}}\right) \mathrm{d} \Gamma_{w k}^{v}\left(1-e^{-\frac{r_{v}^{2}}{4 v_{\infty} t_{v}}}\right)\right] \quad \text { where } \quad r_{v}=\left|\boldsymbol{z}_{w k}^{v}-R_{0} \mathrm{e}^{\imath \theta}\right|
$$

where, $r_{v}$ is indicated in Figure 8, and the effect of viscosity is included inside the integral on the right hand side (RHS). At each time step, the strengths of the vortices that are shed from the edges of the airfoil are determined by imposing a Kutta condition at the TE and a stagnation condition at the LE on the circle in the complex plane. ${ }^{14}$ These constraint conditions on induced velocity were originally derived in Ref. [14] for inviscid flow. Including the effect of viscosity, the modified expressions are as follows. 
The stagnation condition at the LE:

$$
\begin{gathered}
\frac{1}{R_{0}}\left[A_{1}-\left(A_{2}+\frac{1}{2} A_{7}\right)+A_{10}\right]-2 U_{I} \sin \left(\alpha+\alpha_{f s}\right)=\frac{1}{2 \pi R_{0}} \oint_{w k}\left[\mathscr{R}\left(\frac{\boldsymbol{z}_{w k}^{v}-R_{0}}{\boldsymbol{z}_{w k}^{v}+R_{0}}\right) \mathrm{d} \Gamma_{w k}^{v}\left(1-e^{-\frac{r_{v}^{2}}{4 v_{w} t_{v}}}\right)\right] \\
\text { where } \quad r_{v}=\left|\boldsymbol{z}_{w k}^{v}+R_{0}\right|
\end{gathered}
$$

The Kutta condition at the TE:

$$
\Gamma_{0}=-\oint_{w k}\left[\mathscr{R}\left(\frac{\boldsymbol{z}_{w k}^{v}+R_{0}}{\boldsymbol{z}_{w k}^{v}-R_{0}}\right) \mathrm{d} \Gamma_{w k}^{v}\left(1-e^{-\frac{r_{v}^{2}}{4 v_{\infty} t_{v}}}\right)\right] \quad \text { where } \quad r_{v}=\left|\boldsymbol{z}_{w k}^{v}-R_{0}\right|
$$

where, the effect of viscosity is included inside the integrals on the RHS of Eqs. (10) and (11). At each time step, the latest shed vortices are placed at pre-determined distances from the LE and TE, and their inviscid strengths are computed using Eqs. (10) and (11) as described in Ref. [14]. Subsequently, the wake induced vorticity on the circle is given as follows:

$$
\begin{aligned}
\left.\gamma_{w i}(\theta, t)\right|_{\text {viscous }} & =-\frac{\Gamma_{0}}{2 \pi R_{0}}-\frac{1}{2 \pi R_{0}} \oint_{w k}\left[\mathscr{R}\left(\frac{\boldsymbol{z}_{w k}^{v}+R_{0} \mathrm{e}^{\imath \theta}}{\boldsymbol{z}_{w k}^{v}-R_{0} \mathrm{e}^{\imath \theta}}\right) \mathrm{d} \Gamma_{w k}^{v}\left(1-e^{-\frac{r_{v}^{2}}{4 v_{\infty} t_{v}}}\right)\right] \\
\text { where } \quad r_{v} & =\left|\boldsymbol{z}_{w k}^{v}-R_{0} \mathrm{e}^{\imath \theta}\right|
\end{aligned}
$$

\section{Calculation of the bound vorticity}

The airfoil bound vorticity on the circle is obtained by combining Eqs. (4), (6), and (12) as follows.

$$
\gamma_{b}=\gamma_{f s}+\gamma_{u s}^{f l e x}+\left.\gamma_{w i}\right|_{v i s c o u s}
$$

\section{Wake evolution}

Vortices on the flattened NC are convected using the Rott-Birkhoff equation, ${ }^{28}$ which is derived from BiotSavart law for two dimensional flow. The Rott-Birkhoff equation yields induced velocity at any point due to a vortex and it is implemented in the $\xi^{I}-\eta^{I}$ coordinate system as shown in Eq. (14).

$$
\mathbf{q}^{*}\left(\boldsymbol{\zeta}^{I}\right)=\sum_{k=1}^{\infty}\left[\frac{\mathrm{d} \Gamma_{k}}{2 \pi \imath\left(\boldsymbol{\zeta}^{I}-\boldsymbol{\zeta}_{k}^{I}\right)}+\left(u_{E}-\imath v_{E}\right)\right]
$$

where, $\infty$ indicates that the summation includes airfoil bound and all shed vortices in the airfoil-wake system, and $u_{E}, v_{E}$ are components of the free stream velocity at each shed vortex. The effect of free stream velocity due to forward flight is incorporated by using

$$
u_{E}=-u_{\infty} \cos \tilde{\varphi}_{\Gamma_{k}} \quad \text { and } \quad v_{E}=v_{\infty}
$$

where $\tilde{\varphi}_{\Gamma_{k}}$ denotes the instantaneous sweep angle of the $\mathrm{d} \Gamma_{k}$, as depicted in Figure 7. Therefore, Eq. (14) yields

$$
\mathbf{q}^{*}\left(\zeta^{I}\right)=\sum_{k=1}^{\infty}\left[\frac{\mathrm{d} \Gamma_{k}}{2 \pi \imath\left(\zeta^{I}-\zeta_{k}^{I}\right)}+\left(-u_{\infty} \cos \tilde{\varphi}_{\Gamma_{k}}-\imath v_{\infty}\right)\right]
$$

Note that Eq. (16) becomes singular as $\left(\zeta^{I}-\zeta_{k}^{I}\right)$ approaches zero. Therefore, numerical implementation of Eq. (16) requires de-singularization of the vortex core, in which each discrete vortex is assumed to have a finite core radius. In this paper, the de-singularization procedure adopted in Ref. [13] is used. Consequently, Eq. (16) is modified as follows.

$$
\begin{aligned}
\mathbf{q}^{*}\left(\boldsymbol{\zeta}^{I}\right) & =\sum_{k=1}^{\infty}\left[\frac{\mathrm{d} \Gamma_{k}}{2 \pi \imath\left(\zeta^{I}-\zeta_{k}^{I}\right)} \frac{\left(\zeta^{I}-\zeta_{k}^{I}\right)^{*}}{\left(\zeta^{I}-\zeta_{k}^{I}\right)^{*}}+\left(-u_{\infty} \cos \tilde{\varphi}_{\Gamma_{k}}-\imath v_{\infty}\right)\right] \\
& =\sum_{k=1}^{\infty}\left[\frac{\mathrm{d} \Gamma_{k}\left(\zeta^{I}-\boldsymbol{\zeta}_{k}^{I}\right)^{*}}{2 \pi \imath\left\|\zeta^{I}-\boldsymbol{\zeta}_{k}^{I}\right\|^{2}}+\left(-u_{\infty} \cos \tilde{\varphi}_{\Gamma_{k}}-\imath v_{\infty}\right)\right] \\
& \approx \sum_{k=1}^{\infty}\left[\frac{\mathrm{d} \Gamma_{k}\left(\zeta^{I}-\boldsymbol{\zeta}_{k}^{I}\right)^{*}}{2 \pi \imath\left(r_{c}^{2}+\left\|\zeta^{I}-\boldsymbol{\zeta}_{k}^{I}\right\|^{2}\right)}+\left(-u_{\infty} \cos \tilde{\varphi}_{\Gamma_{k}}-\imath v_{\infty}\right)\right]
\end{aligned}
$$


Incorporating the effect of viscosity, Eq. (17) yields

$$
\begin{gathered}
\left.\mathbf{q}^{*}\left(\zeta^{I}\right)\right|_{\text {viscous }} \approx \sum_{k=1}^{\infty}\left[\frac{\mathrm{d} \Gamma_{k}\left(\zeta^{I}-\boldsymbol{\zeta}_{k}^{I}\right)^{*}}{2 \pi \imath\left(r_{c}^{2}+\left\|\zeta^{I}-\zeta_{k}^{I}\right\|^{2}\right)}\left(1-e^{-\frac{r_{v_{k}}^{2}}{4 v_{\infty} t_{k}}}\right)+\left(-u_{\infty} \cos \tilde{\varphi}_{\Gamma_{k}}-\imath v_{\infty}\right)\right] \\
\text { where } r_{v_{k}}=\left|\zeta^{I}-\zeta_{k}^{I}\right|
\end{gathered}
$$

It was noted in Ref. [15] that the use of wake sub-iterations improves quality of the solution, especially when wake distortion due to wing-wake interaction is expected. Therefore, the Euler scheme, which was used in Ref. [15], is used in the current study. In this scheme, the positions of the wake vortices are computed by performing several sub-iterations within each time step. Then,

$$
\boldsymbol{\zeta}^{I}(t+\Delta t)=\zeta^{I}(t)+\sum_{j=1}^{n_{w k s u b i t}} \mathbf{q}_{j}(t) \frac{\Delta t}{n_{w k s u b i t}}
$$

\section{Calculation of Aerodynamic Loads using the Unsteady Bernoulli Equation}

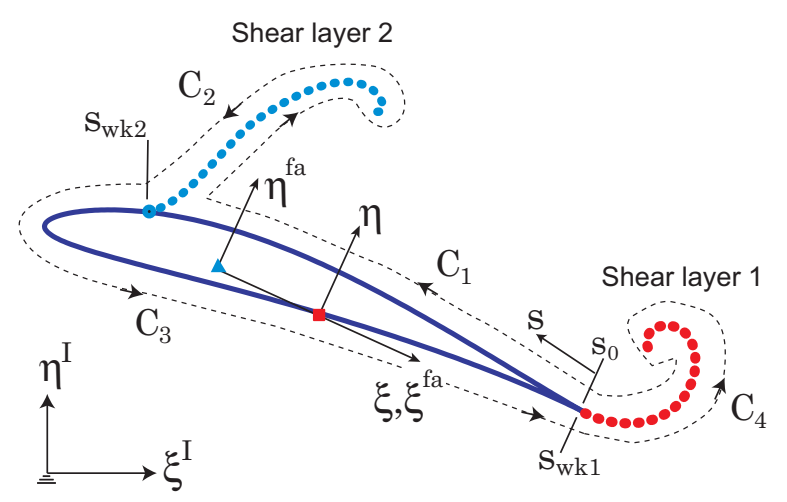

Figure 9. Contours of integration for computing velocity potential from bound and shed vorticity from an airfoil. For clarity, shed vortices are indicated by solid circles.

The unsteady Bernoulli principle relates the local pressure at any point to the velocity potential at that point. The expression derived in the $\xi^{f a}-\eta^{f a}$ coordinate system ${ }^{21}$ is as follows:

$$
\frac{p_{\infty}-p}{\rho_{\infty}}=\frac{\partial \phi}{\partial t}+\frac{1}{2}\left[\left(\frac{\partial \phi}{\partial \xi f a}\right)^{2}+\left(\frac{\partial \phi}{\partial \eta^{f a}}\right)^{2}\right]-\left(v_{\xi}^{f a} \frac{\partial \phi}{\partial \xi f a}+v_{\eta}^{f a} \frac{\partial \phi}{\partial \eta^{f a}}\right)
$$

where $v_{\xi}^{f a}$ and $v_{\eta}^{f a}$ are the velocities of the airfoil resolved in the $\xi^{f a}-\eta^{f a}$ coordinate system. It is important to note that the velocity potential is discontinuous across shear layers, i.e. the wakes shed from the airfoil; therefore, it has to be computed along piecewise continuous contours. ${ }^{29}$ Vorticity and circulation are defined as positive when counter-clockwise; therefore, integration along the contours is performed in a counter-clockwise manner. The origin of integration is selected to be a point close to the trailing edge on the upper surface of the airfoil as shown in Figure 9. Furthermore, let $s_{w k 1}$ and $s_{w k 2}$ denote the arc coordinates at which the shear layers are formed, shown in Figure 9, and these denote the location at which $\phi$ is discontinuous. For an airfoil with a sharp trailing edge, a shear layer emanates from the trailing edge; therefore, $s_{w k 1}=L_{C}$, and the total velocity potential at any point in the airfoil wake system is given by

$$
\phi(s, t)=\phi_{b}(s, t)+\phi_{w k 1}(s, t)
$$

For attached flow, $\phi_{b}$ is obtained by integrating the vorticity along contour $C_{1}$ shown in Figure 9 . For separated flow, $\phi_{b}$ is obtained by integrating the vorticity along contours $C_{1}-C_{2}-C_{3}$. In Ref. [21], the expressions of $\phi_{b}$ and $\phi_{w k 1}$ were obtained for inviscid flow. Incorporating the effect of viscosity, the modified expressions are as follows: 
For $0<s<s_{w k 2}$

$$
\phi_{b}(s, t)=\phi_{0}+\int_{0}^{s} \gamma_{b} d s
$$

For $s_{w k 2}<s<L_{C}$

$$
\phi_{b}(s, t)=\phi_{0}+\int_{0}^{s_{w k 2^{-}}} \gamma_{b} d s+\int_{L_{w k 2}}\left\{\gamma_{w k 2}(\varsigma, t)\left(1-e^{-\frac{r_{v}^{2}}{4 v_{\infty} t_{v}}}\right)\right\} \mathrm{d} \varsigma+\int_{s_{w k 2^{+}}}^{s} \gamma_{b} d s
$$

The velocity potential due to shear layer 1 is given by

$$
\phi_{w k 1}(s, t)=\int_{L_{w k 1}}\left\{\frac{\gamma_{w k 1}(\varsigma, t)}{2 \pi} \varphi(\varsigma, s, t)\left(1-e^{-\frac{r_{v}^{2}}{4 v_{\infty} t_{v}}}\right)\right\} \mathrm{d} \varsigma
$$

where, in Eqs. (22) and (23), the effect of viscosity is included inside the integrals on the RHS and

$$
r_{v}=\left|\zeta^{v, f a}(\varsigma, t)-\zeta^{f a}(s)\right|
$$

Moreover $\varphi \in[0,2 \pi)$, depicted in Figure 8, is given by

$$
\varphi(\varsigma, s, t)=\operatorname{argument}\left(\zeta^{v, f a}(\varsigma, t)-\zeta^{f a}(s)\right)
$$

\section{Fluid-structure coupling}
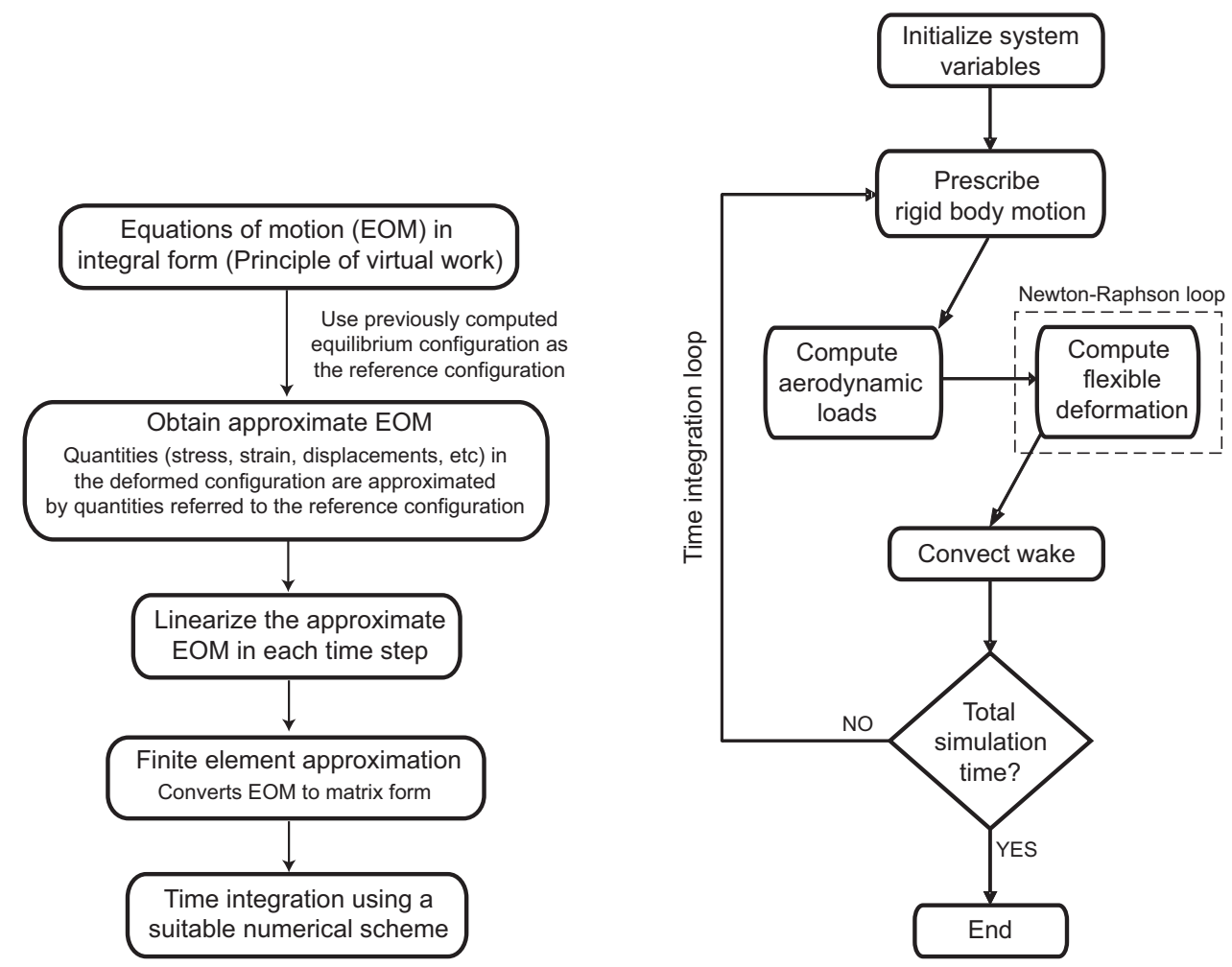

Figure 10. Formulation of the aeroelastic equations (left) and implementation of the aeroelastic model in MARC (right).

The equations of motion representing the aeroelastic response problem are obtained from an updated Lagrangian (UL) approach. ${ }^{30,31}$ An approximate solution is obtained by referring all the quantities (stress, strain and displacements) of the deformed configuration to the equilibrium configuration obtained in the previous time step, and linearizing the resulting equations of motion (EOM). Implementation of the UL formulation in 
MARC $^{30,31}$ is illustrated by Figure 10 and was summarized in Ref. [20]. The implementation of the coupled problem is shown in the block diagram given in Figure 10. At each time step, rigid body motion is prescribed as displacements at specified nodes. The aerodynamic loads, computed based on the wing motion at the beginning of each time step, are applied to the structure via FORCEM, a user defined subroutine in MARC. This subroutine is called from the main program for each step of the Newton-Raphson iteration within a time step to ensure convergence of the structural displacements for the applied loads. Finally, the vortices shed into the wake are convected at the end of the time step.

\section{Results and Discussion}

The results are presented for rigid and flexible configurations: (1) aerodynamic comparisons for rigid airfoils and wings in hover and forward flight, and (2) aeroelastic results for anisotropic wings in hover and forward flight.

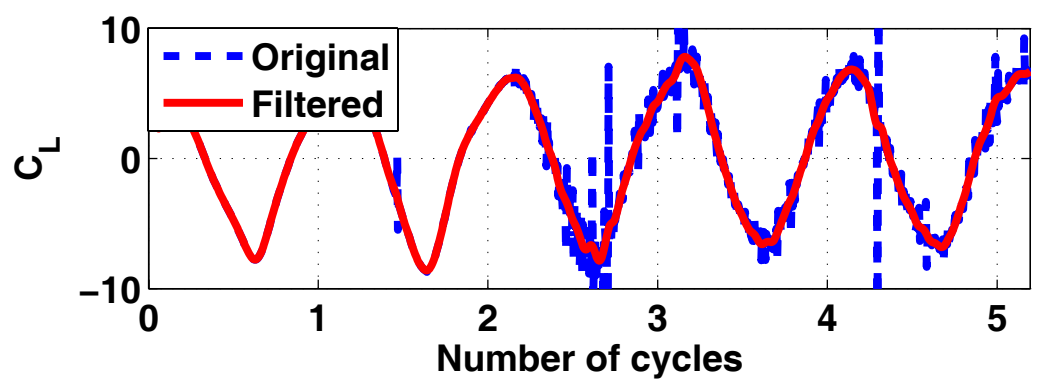

Figure 11. Sample comparison of original and filtered load signals

When using the approximate aerodynamic model, leading edge separation is assumed unless specified otherwise and the transient aerodynamic loads are obtained using the unsteady Bernoulli equation. A previous study ${ }^{21}$ noted that the interaction of the airfoil with previously shed vortices generated large amplitude numerical oscillations in the aerodynamic loads that had to be eliminated before the unsteady loads can be applied on the flexible structure in aeroelastic simulations. The numerical oscillations are not critical to simulations involving rigid airfoils and wings; however, smoothing the signals improves clarity when comparing the time histories of the aerodynamic loads with CFD based results. In this paper, the load signals, which are obtained from the approximate aerodynamic model for rigid cases as well as the flexible cases, are postprocessed using zero-phase digital filters available in MATLAB $^{\circledR}$ (version 8.0). The filters are implemented in MATLAB $^{\circledR}$ using the filtfilt command as shown in Eq (26). A sample comparison of the original and smoothed signals is shown in Figure 11.

$$
y_{\text {filtered }}=\operatorname{filtfilt}\left(B_{f}, A_{f}, y_{\text {unfiltered }}\right)
$$

where $B_{f}$ and $A_{f}$ are filter coefficients based on Chebyshev functions that are arrays of length $\left(m_{f}+1\right)$. The implementation of the zero-phase digital filters may be obtained from the extensive documentation provided in MATLAB $^{\circledR}$.

\section{A. Aerodynamic Comparisons}

Results are presented for the following cases: (1) flat plate airfoils undergoing prescribed motion, (2) rigid Zimmerman wings in hover, and (3) rigid Zimmerman wings in forward flight.

\section{Airfoil Cases}

The forces generated by airfoils undergoing prescribed motion are presented in Figures 12 and 13 for the case of hover, whereas Figure 14 includes a free stream velocity and therefore corresponds to the case of forward flight. The lift and drag are components of the aerodynamic force along the $\eta^{I}$ and $\xi^{I}$ axes respectively. The airfoil kinematics are described by Eq. (27) and the parameters considered are shown in Table 1.

$$
\begin{aligned}
l(t) & =l_{0} \sin (2 \pi f t) \\
\alpha(t) & =\frac{\pi}{2}+\alpha_{0} \sin \left(2 \pi f t+\varphi_{\alpha}\right)
\end{aligned}
$$




\begin{tabular}{|c|l|c|c|c|c|}
\hline Case ID & Name & $f, \mathrm{~Hz}$ & $l_{0}$ & $\alpha_{0}$ & $\varphi_{\alpha}$ \\
\hline 1 & Delayed rotation & $\frac{1}{2 \pi}$ & $c$ & $\frac{\pi}{4}$ & $\frac{\pi}{3}$ \\
\hline 12 & Synchronized rotation & $\frac{1}{3 \pi}$ & $1.5 c$ & $\frac{4 \pi}{9}$ & $\frac{\pi}{2}$ \\
\hline
\end{tabular}

Table 1. Amplitudes and phase for airfoil kinematics. The case ids are obtained from Ref. [32]

where, the pitching is about the mid-chord. The results are obtained for $R e=100, c=1 \mathrm{~m}, \rho_{\infty}=1 \mathrm{~kg} / \mathrm{m}^{3}$, $v_{\infty}=0.01 \mathrm{~m}^{2} / \mathrm{s}, U_{r e f}=1.0 \mathrm{~m} / \mathrm{s}$, where $R e$ and $U_{r e f}$ are defined as follows.

$$
R e=\frac{U_{r e f} c}{v_{\infty}} \quad \text { and } \quad U_{r e f}=2 \pi f l_{0}
$$

The non-dimensional forces are defined as follows:

$$
C_{L}=\frac{L}{\frac{1}{2} \rho_{\infty} U_{r e f}^{2}} \quad \text { and } \quad C_{D}=\frac{D}{\frac{1}{2} \rho_{\infty} U_{r e f}^{2} c}
$$

The CFD based results are taken from Ref. [32]. The approximate results were obtained for the following parameters: $N_{\theta}=200, r_{c}=0.1 c, n_{w k s u b i t}=4$, and circulation limit ${ }^{21}$ was fixed at 2.0. For both cases, CFD simulations indicated that there was leading edge separation. However, for case 12 , the vorticity shed from the LE was substantially weaker when compared to that shed from the TE. Therefore, the approximate model was also used with the attached flow assumption. The simulations using the approximate model were conducted assuming both inviscid as well as viscous flow.
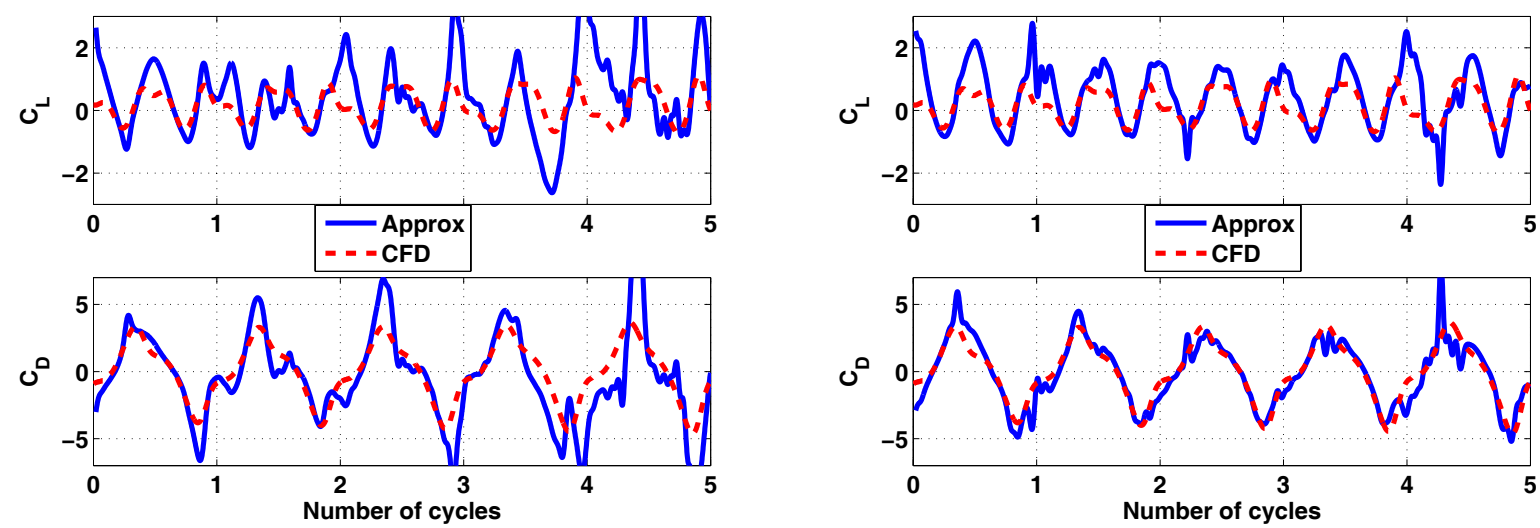

(a) Inviscid separated flow

(b) Separated flow with viscous effects

Figure 12. Force coefficients for Case 1

The force coefficients obtained for case 1 are shown in Figure 12; results obtained by assuming inviscid flow and incorporating viscous effects are shown in Figures 12(a) and 12(b) respectively. Simulations using the approximate model were coducted using 500 time steps per cycle, and the load signals were smoothed using the filter described in Table 2. The comparisons illustrate that incorporating the effect of viscosity improves correlation with CFD based results; in particular, the improvement is noticeable for $C_{D}$. These results suggest that the influence of fluid viscosity in the interactions involving shed vorticity is important for this case.

The force coefficients obtained for case 12 are shown in Figure 13. Simulations assuming separated and attached flows were conducted by using 800 and 500 time steps per cycle respectively, and the loads obtained were smoothed using filters described in Tables 2 and 3 respectively. The results for separated flow, shown in Figures 13(a) and 13(b), indicate that the inviscid approximate model does not compare well with CFD for this case. Incorporating viscous effects improves correlation slightly, as shown in Figure 13(b). However, the 

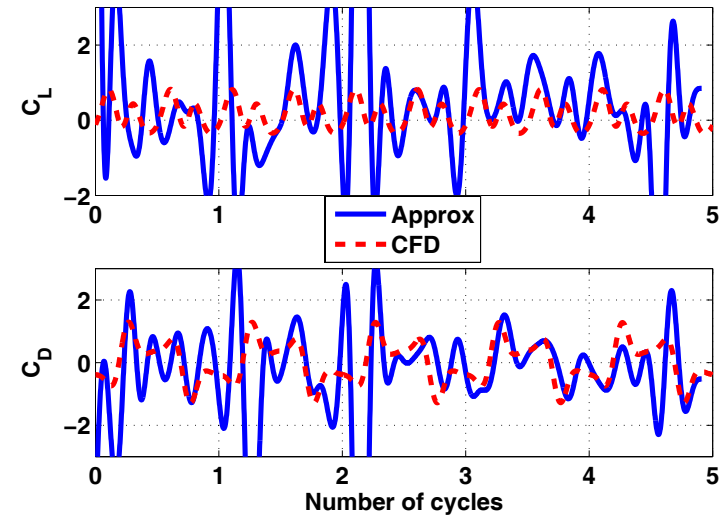

(a) Inviscid separated flow
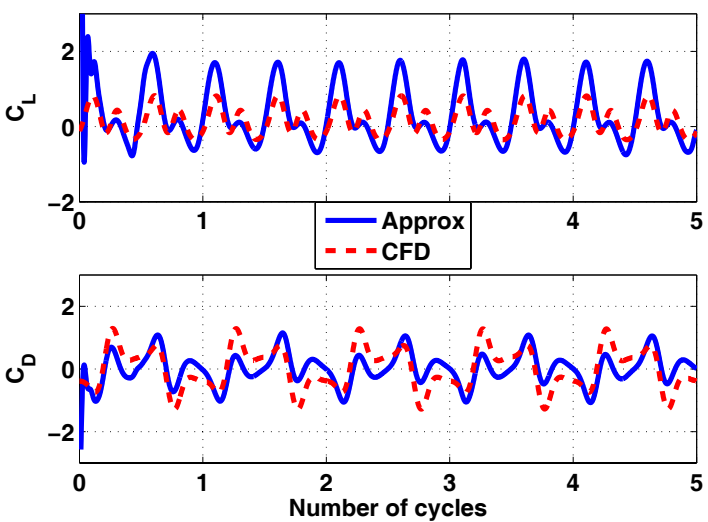

(c) Inviscid attached flow
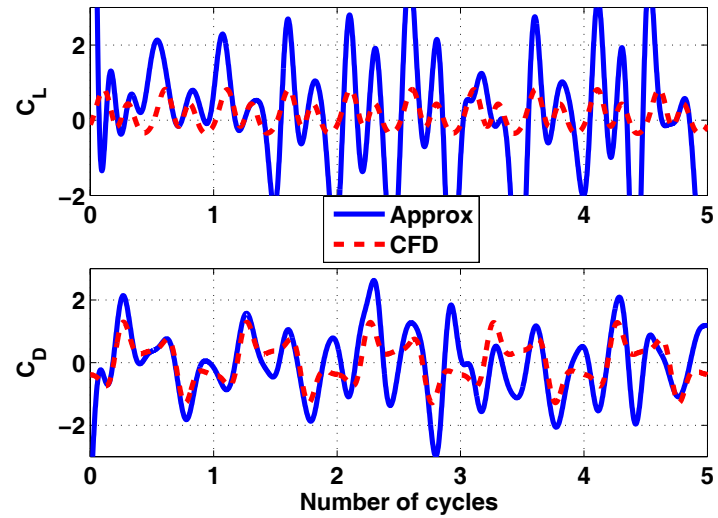

(b) Separated flow with viscous effects
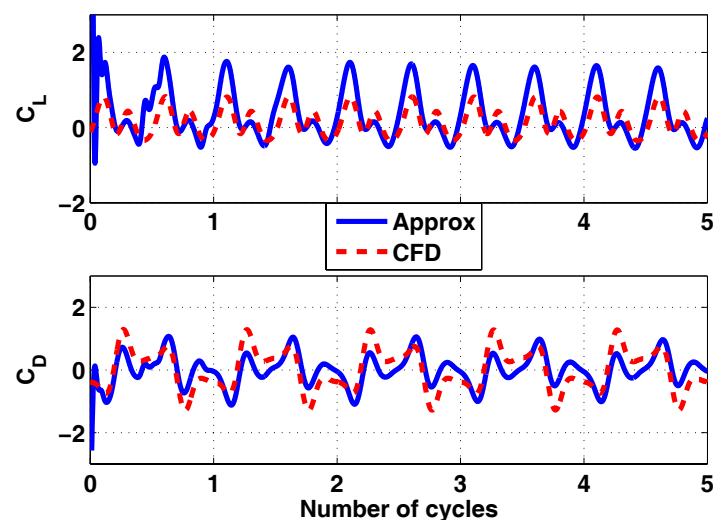

(d) Attached flow with viscous effects

Figure 13. Force coefficients for Case 12

improvement is most noticeable in the first and second cycles of $C_{D}$. Results obtained by assuming attached flow, shown in Figures 13(c) and 13(d), indicate that the approximate results show reasonable correlation with CFD based results; however some of the peaks in $C_{D}$ are not adequately captured. Incorporating viscous effects improves correlation slightly; however, the difference is small. These results imply that when the vorticity generated from the $\mathrm{LE}$ is weak, the principal contribution to the aerodynamic loads is from the TE vortices.

The forces generated by the airfoils in the presence of a free stream are shown in Figure 14. The kinematics employed are the same as those for cases 1 and 12, where a free stream velocity vector that is parallel to $\xi^{I}$ is now introduced; the free steam velocity is equal to $0.2 U_{r e f}$, where $U_{r e f}$ is given in Eq. (29). Simulations with the approximate model were carried out assuming separated flow for case 1, attached flow for case 12, and including effect of viscosity. The results were obtained using 500 time steps per cycle, and smoothed using the filter described in Table 2. Comparisons indicate that the approximate model produces reasonable agreement with CFD for the cases considered. In particular, comparisons for case 12, shown in Figure 14(b), indicate that the discrepancy in the peaks of $C_{D}$ is larger when compared to the discrepancy observed for case of hover; this may be attributed to the increased contribution of vorticity shed from the LE. 


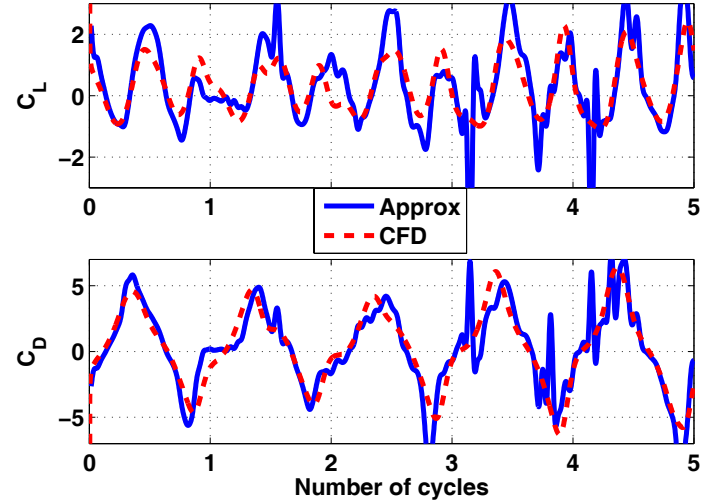

(a) Case 1: separated flow with viscous effects
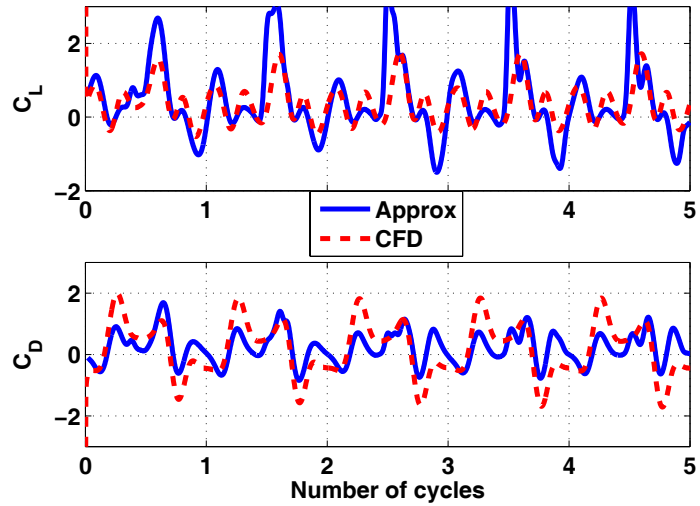

(b) Case 12: attached flow with viscous effects

Figure 14. Force coefficients for Cases 1 and 12 with free stream

\begin{tabular}{|c|c|c|c|c|c|}
\hline$A_{f}$ & 1.0000 & -3.5379 & 4.7367 & -2.8414 & 0.6439 \\
\hline$B_{f}\left(\times 10^{-3}\right)$ & 0.0797 & 0.3186 & 0.4779 & 0.3186 & 0.0797 \\
\hline
\end{tabular}

Table 2. Filter coefficients

\section{Rigid Wings in Hover}

The results for the rigid wings are based on a Zimmerman planform, shown in Figure 2, that have an aspect ratio of $7.65, c_{r}=25 \mathrm{~mm}, R_{\text {span }}=75 \mathrm{~mm}$ respectively. ${ }^{33}$ The CFD simulations were conducted using the numerical framework described in Ref. [34,35] that solves the finite-volume-based Navier- Stokes equations using a pressure-based algorithm. The aerodynamic loads obtained using the approximate aerodynamic model were computed for the following set of parameters: $N_{\text {sections }}=59, N_{\theta}=100, r_{c}=0.1 \times$ chord, and $n_{w k s u b i t}=4$ respectively. The numerical experiments were conducted in air $\left(\rho_{\infty}=1.209 \mathrm{~kg} / \mathrm{m}^{3}, v_{\infty}=1.568 \times 10^{-5} \mathrm{~m}^{2} / \mathrm{s}\right)$.

The lift and thrust generated by rigid wings are shown in Figures 15, 16, and 17. The lift and thrust are the components of the aerodynamic force resolved along $Y_{S P}$ and $Z_{S P}$, shown in Figure 4, respectively. The corresponding non-dimensional quantities are defined in Eq. (30).

$$
C_{L}=\frac{L}{\frac{1}{2} \rho_{\infty} U_{r e f}^{2} R_{\text {span }} c_{r}} \quad \text { and } \quad C_{T}=\frac{T}{\frac{1}{2} \rho_{\infty} U_{r e f}^{2} R_{\text {span }} c_{r}} \quad \text { where } \quad U_{r e f}=4 \pi \beta_{0} f
$$

Furthermore, $X_{W}$ and $Y_{W}$, shown in Figure 2, coincide with $X_{S P}$ and $Z_{S P}$, shown in Figure 5, respectively at the start of the motion. The flapping motion, which corresponds to a simple rotation about $Y_{W}$, given by Eq. (31).

$$
\beta(t)=\beta_{0} \sin (2 \pi f t)
$$

The non-dimensional lift and thrust for $\beta_{0}=35^{\circ}$ and various values of $f$ are shown in Figures 15 and 16 . Simulations using the approximate and CFD based models were conducted using 300 and 500 time steps per flapping cycle to discretize the motion respectively. Subsequently, a fourth order filter $\left(m_{f}=4\right)$ described in Table 4 was used to smooth the signals obtained using the approximate model.

Figure 15 shows the force coefficients obtained for $\beta_{0}=35^{\circ}$ and $f=10 \mathrm{~Hz}$. The result indicates that the approximate model over-predicts the lift compared to the CFD based result; the mean and maximum errors in the peaks are $50 \%$ and $100 \%$ respectively. The discrepancy may be attributed to the spanwise flow and tip vortices that may be important for the combination of amplitude and flapping frequencies that are considered, which are not incorporated in the approximate aerodynamic model. Due to the lack of wing pitch or twist, the thrust corresponds to aerodynamic force parallel to the chordwise direction of the wing. In the approximate computations, the wing is assumed to have zero thickness; consequently, the force parallel to the wing chord is 


\begin{tabular}{|c|c|c|c|c|c|}
\hline$A_{f}$ & 1.0000 & -3.9109 & 5.7376 & -3.7423 & 0.9156 \\
\hline$B_{f}\left(\times 10^{-6}\right)$ & 0.1517 & 0.6067 & 0.9101 & 0.6067 & 0.1517 \\
\hline
\end{tabular}

Table 3. Filter coefficients

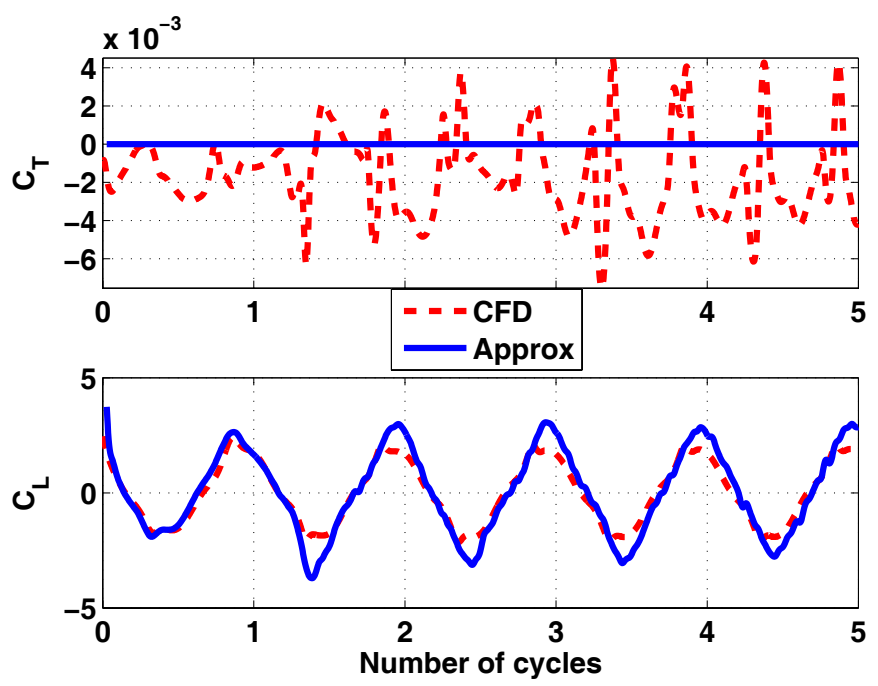

Figure 15. Force coefficients generated by rigid wings for $35^{\circ}$ and $f=10 \mathrm{~Hz}$

equal to zero. In the CFD based computations, this quantity is equal to the sum of viscous forces on the wing surface and the suction pressure on the edges of the wing; the result in Figure 15 indicates that the thrust obtained from CFD based calculations is several orders of magnitude lower than the lift.

The time histories of $C_{L}$ for all the flapping frequencies considered is shown in Figure 16, wherein the CFD based and approximate results are shown on separate plots. The thrust was several orders of magnitude lower than lift; consequently comparisons involving this quantity are not shown. It is interesting to note that the lift coefficients, computed for various frequencies, are very similar. This trend indicates that $C_{L}$ is somewhat insensitive to Reynolds number. Furthermore, based on the definition of $C_{L}$ in Eq. (30), it follows that the peak lift is proportional to the square of the flapping frequency. Similar trends are obtained from simulations using the approximate model for $\beta_{0}=18^{\circ}$, as shown in Figure 17, indicating that the relation between the lift and flapping frequency is insensitive to flapping amplitude.

\begin{tabular}{|c|c|c|c|c|c|}
\hline$A_{f}$ & 1 & -3.0456 & 3.6243 & -1.9780 & 0.4158 \\
\hline$B_{f}$ & 0.0010 & 0.0041 & 0.0062 & 0.0041 & 0.0010 \\
\hline
\end{tabular}

Table 4. Filter coefficients

The forces generated by rigid wings undergoing prescribed combined flap-pitch motion are shown in Figure 18. The wing kinematics are described by the following Euler rotations: a flapping rotation about $Y_{W}$ described by Eq. (31) followed by a feathering motion about $X_{W}$ described by Eq. (32); where, $X_{W}$, indicated in Figure 2 , originates at the quarter-chord point of the root. The parameters for which the results are obtained are summarized in Table 5. The CFD and approximate simulations were conducted by using 500 and 200 time steps per flapping cycle respectively. The results, in Figure 18, indicate that the approximate model shows reasonable correlation with CFD for the cases considered.

$$
\alpha(t)=-\alpha_{0} \sin (2 \pi f t)
$$

\section{3. $\quad$ Rigid Wings in Forward Flight}

The forces generated by rigid wings in forward flight, shown in Figures 20 and 21, are obtained for combinations of $\mu$ and $\beta_{s p}$ that are based on experimentally obtained data for hawkmoths in forward flight. ${ }^{27}$ The 


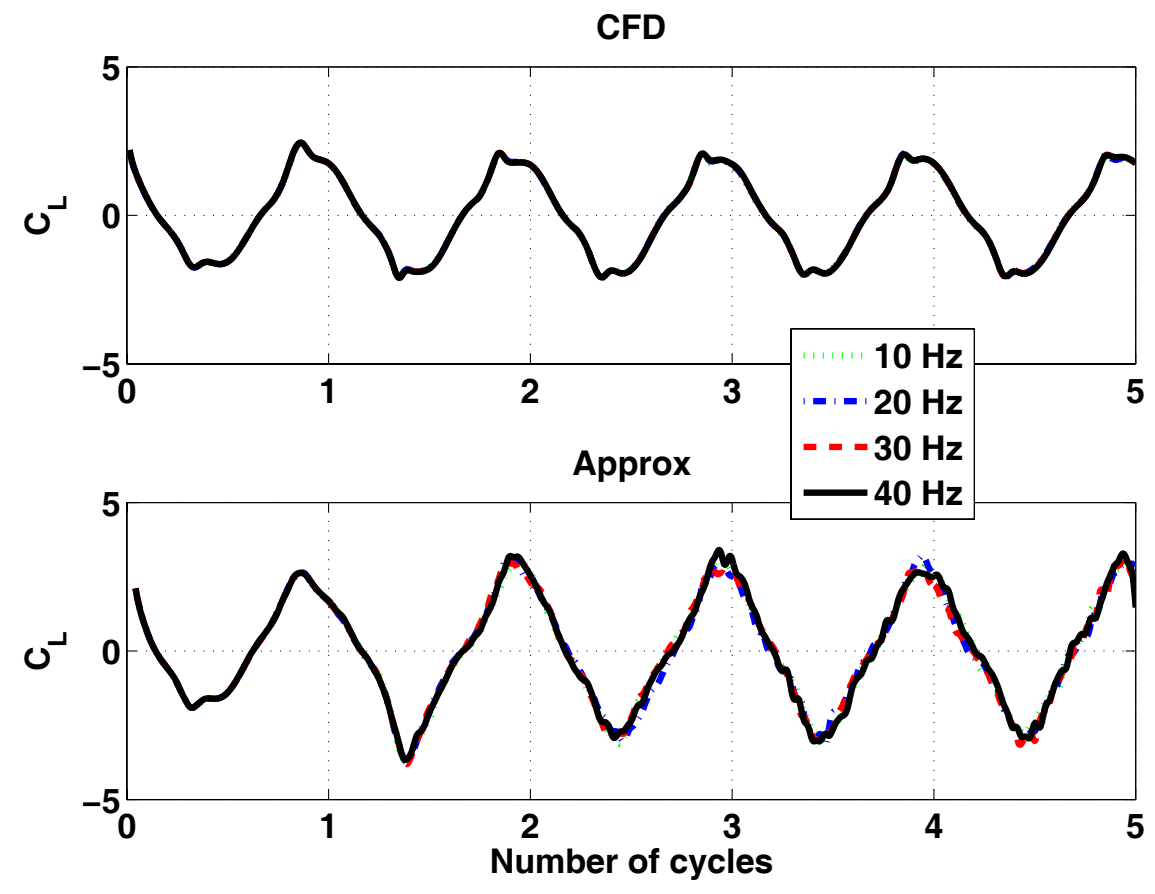

Figure 16. Lift coefficients generated by rigid wings for $\beta_{0}=35^{\circ}$.

\begin{tabular}{|c|c|c|c|}
\hline Figure Number & Flap amplitude & Pitch amplitude & $f$ \\
\hline $18(\mathrm{a})$ & $10^{\circ}$ & $5^{\circ}$ & $10 \mathrm{~Hz}$ \\
$18(\mathrm{~b})$ & $15^{\circ}$ & $5^{\circ}$ & $10 \mathrm{~Hz}$ \\
$18(\mathrm{c})$ & $15^{\circ}$ & $10^{\circ}$ & $10 \mathrm{~Hz}$ \\
\hline
\end{tabular}

Table 5. Force coefficients generated by wings undergoing combined flap-pitch rotation.

specific combinations used in the current study, which are based on the data obtained for Moth M1 in Ref. [27], are shown in Figure 19 and listed in Table 6. The components of free stream velocity are calculated using Eq. (1) where $U_{t i p}=2 \pi \beta_{0} f R_{\text {span }}$.

\begin{tabular}{|c|c|c|c|c|c|c|}
\hline$\mu$ & 0 & 0.05 & 0.10 & 0.15 & 0.20 & 0.25 \\
\hline$\beta_{s p}$ & $0^{\circ}$ & $14^{\circ}$ & $21.2^{\circ}$ & $27.7^{\circ}$ & $34^{\circ}$ & $40^{\circ}$ \\
\hline
\end{tabular}

Table 6. Combination of advance ratios and stroke plane inclinations used in the current study

The non-dimensional lift generated by rigid wings for $\beta_{0}=35^{\circ}, f=10 \mathrm{~Hz}$, and various advance ratios, are shown in Figure 20. The thrust generated was several orders of magnitude lower than lift; therefore results for this quantity are not presented. The CFD and approximate simulations were conducted by using 500 and 300 time steps per flapping cycle respectively; results obtained using the approximate model were smoothed using the filter described in Table 4. The comparisons indicate that the approximate model shows reasonable agreement with CFD based results for the cases considered. The correlation between the results improves with increasing advance ratio. The mean lift coefficients, which represent the time averaged values of $C_{L}$, are shown in Figure 21. This result shows that the mean lift decreases (becomes more negative) with increasing forward flight speed, and that the approximate model over-predicts the value but captures the trend. The results shown in Figures 20 and 21 demonstrate that the modified aerodynamic model can be used to conduct trend type studies for wings in forward flight despite the simplifying assumptions in the formulation. 


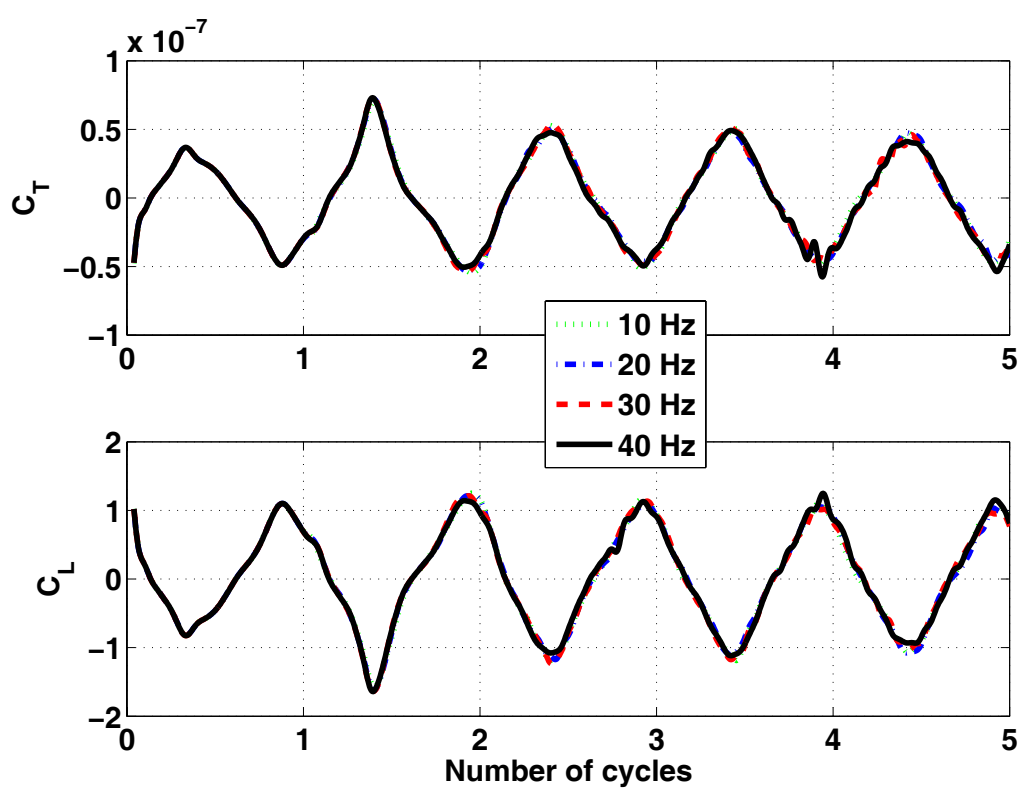

Figure 17. Lift and thrust coefficients, calculated using the approximate model, generated by rigid wings for $\beta_{0}=18^{\circ}$.

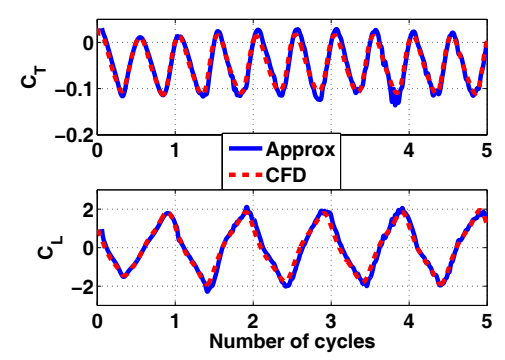

(a) $\beta_{0}=10^{\circ}, \alpha_{0}=5^{\circ}$

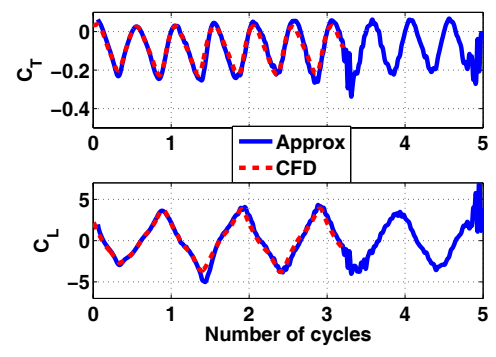

(b) $\beta_{0}=15^{\circ}, \alpha_{0}=5^{\circ}$

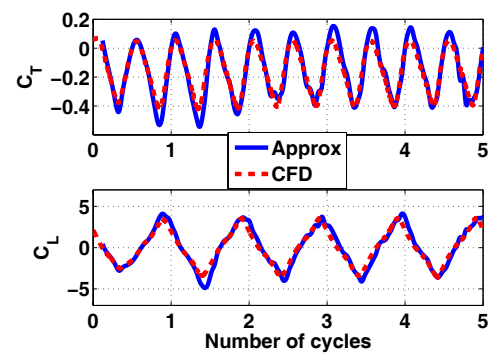

(c) $\beta_{0}=15^{\circ}, \alpha_{0}=10^{\circ}$

Figure 18. Force coefficients generated by a rigid wing undergoing combined pitch-flap motion.

\section{B. Aeroelastic Results}

Aeroelastic simulations were conducted using anisotropic wings that are based on an unstressed CAPRAN film (membrane) that is supported by a carbon fiber based spar-batten skeleton. Following the notation used in Ref. [33], the wings are labeled as LiBj where $i$ and $j$ denote the number of prepreg layers in the LE spar and the battens, as shown in Figure 22. Structural dynamic modeling of the anisotropic wings in MARC was presented in Ref. [16]. The finite element models of the wings, shown in Figure 2, are composed of 1263 shell elements (Element type 75 in MARC) that are capable of modeling large rigid body motion as well as moderate-to-large flexible deformation. The geometric and material properties of the prepreg and the membrane ${ }^{16}$ are provided in Table 7. Wing kinematics are implemented as displacement boundary conditions at the nodes that form the triangular root. Furthermore, the EOM obtained using the UL method are integrated forward in time using a single step Houbolt numerical scheme. ${ }^{36}$

During the simulations, a pressure based filter that is described by Eq. (33) was used to limit the magnitude of numerical noise that is transmitted to the flexible wing; note that the limit should be sufficiently high 


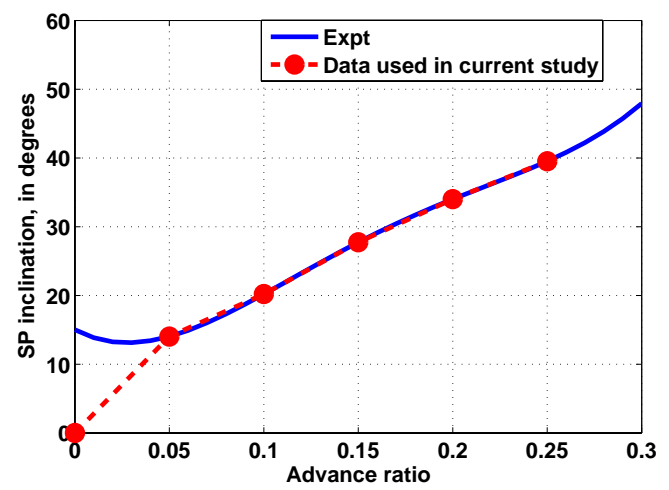

Figure 19. Combinations of advance ratio and SP inclination: Expt - Experimental data. ${ }^{27}$

so as not to introduce significant errors during calculations.

$$
p_{\text {applied }}= \begin{cases}-p_{\text {limit }} & \text { if } p_{\text {calculated }} \leq-p_{\text {limit }} \\ p_{\text {calculated }} & \text { if }-p_{\text {limit }} \leq p_{\text {calculated }} \leq p_{\text {limit }} \\ p_{\text {limit }} & \text { if } p_{\text {calculated }} \geq p_{\text {limit }}\end{cases}
$$

For all the cases considered in this study, a limiting pressure given by $p_{\text {limit }}=36 p_{r e f}$, where $p_{\text {ref }}$ as defined in Eq. (34), was found to be adequate.

$$
p_{\text {ref }}=\frac{1}{2} \rho_{\infty} U_{t i p}^{2} \quad \text { where } \quad U_{t i p}=2 \pi f \beta_{0} R_{\text {span }}
$$

\begin{tabular}{|c|c|}
\hline & Material Properties \\
\hline Carbon fiber prepreg & $E_{11}=233 \mathrm{GPa}$ \\
& $E_{22}=23.1 \mathrm{GPa}$ \\
(Properties of one layer) & $E_{12}=15.5 \mathrm{GPa}$ (L1B1, L1B2) \\
& $E_{12}=10.5 \mathrm{GPa}$ (all other configs) \\
& $v_{12}=0.05$ \\
& $\rho=1740 \mathrm{~kg} / \mathrm{m}^{3}$ \\
& Thickness $=0.1 \mathrm{~mm}$ \\
\hline Capran membrane & $E=2.76 \mathrm{GPa}$ \\
(From experiments) & $v_{12}=0.489$ (Incompressible) \\
& $\rho=1384 \mathrm{~kg} / \mathrm{m}^{3}$ \\
& Thickness $=15 \mathrm{microns}$ \\
\hline
\end{tabular}

Table 7. Material properties of the composite and membrane

\section{Anisotropic Wings in Hover}

The computed and experimentally measured magnitudes of thrust generated by anisotropic wings are compared. The results, which are obtained for one-layer batten configurations and $\beta_{0}=35^{\circ}$, for a range of flapping frequencies, are shown in Figures 23 and 24. The experimental results were obtained from Ref. [33] and computations are based on the approximate aeroelastic model. Note that the experiments were conducted using two wings undergoing symmetric flapping motion, whereas the computations were performed by considering a single wing; therefore, the thrust obtained from the simulations was multiplied by a factor of two to allow comparison with the experimental result. For the approximate model, 300 time steps per flapping cycle were used to discretize the motion. The simulations, conducted for a total of 5 cycles, indicated that an approximate 
steady state was achieved after two cycles. Therefore, the mean thrust was calculated by time averaging the thrust generated over cycles 2 through 5.

The thrust predicted using the approximate aeroelastic model shows reasonable agreement with experimentally measured thrust for all the cases considered. An accurate assessment of the error is complicated because due to the limited amount of experimental data available, the sensitivity of the thrust to variability in material, geometric properties, and wing construction cannot be determined. Therefore, the effect of differences in structural dynamic modeling on the thrust generated has to be determined before quantifying the differences in the aeroelastic results.

Figure 24 shows the thrust generated by one-layer batten configurations. These results indicate that the approximate model predicts the trends that are observed from the experimental measurements. Different wing configurations produce maximum thrust at different ranges of flapping frequencies. Configuration L1B1 produces maximum thrust at frequencies between $5 \mathrm{~Hz}$ and $18 \mathrm{~Hz}, \mathrm{~L} 2 \mathrm{~B} 1$ for frequencies between $18 \mathrm{~Hz}$ and $35 \mathrm{~Hz}$, and L3B1 for frequencies between $35 \mathrm{~Hz}$ and $40 \mathrm{~Hz}$ respectively.

The thrust generated by L1Bj configurations is shown in Figure 23(a). Experimental measurements as well as the computations for L1B1 show a peak in thrust for a flapping frequency in the range of $20-25 \mathrm{~Hz}$; the computations for L1B2 show a similar peak. In Ref. [16], the fundamental frequency of L1B1 was found to be approximately $21 \mathrm{~Hz}$. Therefore, it appears that the peaks in Figure 23(a) occur when the frequency of wing excitation is close to the fundamental frequency of the wing. Additional simulations using L1B1 were conducted in order to examine the dependence of the peak in thrust on the flapping amplitude. The results, shown in Figure 25, confirm that a peak in thrust is obtained when the excitation frequency is close to the natural frequency of the wing for all the amplitudes considered. This is an observation that might be valuable for wing design and selection of wing kinematics.

The aerodynamic and inertia loads acting on the wings were compared. Sample results for a limited number of cases are shown in Figures 26 and 27. The aerodynamic and inertia loads are calculated using Eq. (35). These results, which resemble similar comparisons for other cases, indicate that the aerodynamic loads are comparable to inertia loads. This is contrary to the observations of Refs. $[10,17,18]$ and suggests that the relative importance of aerodynamic to inertia loads is configuration dependent, thereby highlighting the importance of aeroelastic effects in flapping wings.

$$
\left.\boldsymbol{F}_{s p}\right|_{\text {aero }}=\iint_{A_{w}} p \boldsymbol{d} \boldsymbol{A}_{s p} \quad \text { and }\left.\quad \boldsymbol{F}_{s p}\right|_{\text {inertia }}=\iiint_{V_{w}} d m \ddot{\boldsymbol{x}}_{s p}
$$

\section{Wings undergoing insect-like kinematics for the case of hover}

Results were obtained for rigid and flexible wings that are actuated using insect-like kinematics. The kinematics, based on the hover kinematics of hawkmoths ${ }^{27}$ are obtained by scaling the stroke amplitudes by a factor of two. The time dependent rotations, prescribed about the $Y_{w}, X_{w}$, and $Z_{w}$ axes respectively, are described by the Euler angles in Eq. (36) and shown in Figure 28.

$$
\begin{aligned}
& \Phi_{r}(t)=\frac{\Phi_{0}}{4}+\frac{1}{2} \sum_{j=0}^{3}\left[\Phi_{c j} \cos (2 \pi j f t)+\Phi_{s j} \sin (2 \pi j f t)\right] \\
& \alpha_{r}(t)=-\frac{\alpha_{0}}{4}-\frac{1}{2} \sum_{j=0}^{3}\left[\alpha_{c j} \cos (2 \pi j f t)+\alpha_{s j} \sin (2 \pi j f t)\right] \\
& \Theta_{r}(t)=\frac{\Theta_{0}}{4}+\frac{1}{2} \sum_{j=0}^{3}\left[\Theta_{c j} \cos (2 \pi j f t)+\Theta_{s j} \sin (2 \pi j f t)\right]
\end{aligned}
$$

where $\alpha_{r 1}$ and $\Theta_{r}$ are prescribed about the quarter-chord point at the root. The fourier coefficients of the Euler angles are given in Table 8. The simulations were conducted using 300 time steps per flapping cycle, and the results obtained from the aeroelastic model were smoothed using a filter described by Eq. (2).

The lift and thrust, in grams, generated by rigid and anisotropic wings for $f=10 \mathrm{~Hz}$, and $f=20 \mathrm{~Hz}$, are shown in Figure 29. The results correspond to the forces generated by an isolated flapping wing. For $f=10$ $\mathrm{Hz}$, L1B1 generates higher thrust compared to the other wings, as shown in Figure 29(a); however, the lift generated by all the configurations is somewhat similar. For $f=20 \mathrm{~Hz}$ the results in Figure 29(b) indicate that L1B1 generates less thrust and lift compared to L3B1 and the rigid wing whereas L3B1 produces the 


\begin{tabular}{|c|c|c|c|c|c|c|}
\hline$\Phi_{0}$ & $\Phi_{c 1}$ & $\Phi_{c 2}$ & $\Phi_{c 3}$ & $\Phi_{s 1}$ & $\Phi_{s 2}$ & $\Phi_{s 3}$ \\
\hline 8.1074 & 52.9298 & 0.5959 & 2.2231 & -6.9385 & -2.6643 & 1.5011 \\
\hline
\end{tabular}

\begin{tabular}{|c|c|c|c|c|c|c|}
\hline$\Theta_{0}$ & $\Theta_{c 1}$ & $\Theta_{c 2}$ & $\Theta_{c 3}$ & $\Theta_{s 1}$ & $\Theta_{s 2}$ & $\Theta_{s 3}$ \\
\hline-1.9395 & 1.4152 & 6.1994 & 0.4469 & 2.4752 & 0.5730 & -0.7162 \\
\hline
\end{tabular}

\begin{tabular}{|c|c|c|c|c|c|c|}
\hline$\alpha_{0}$ & $\alpha_{c 1}$ & $\alpha_{c 2}$ & $\alpha_{c 3}$ & $\alpha_{s 1}$ & $\alpha_{s 2}$ & $\alpha_{s 3}$ \\
\hline 9.6698 & -14.9886 & 0.5099 & -0.9282 & -58.9230 & -0.2521 & -7.6834 \\
\hline
\end{tabular}

Table 8. Fourier coefficients in the hawkmoth kinematics. Values are given in degrees.

maximum thrust. Recall that for this frequency, the L1B1 and L3B1 configurations generated the maximum and minimum thrust when using a simple flap actuation. This suggests that the impact of wing flexibility may be kinematics dependent.

\section{Anisotropic Wings in Forward Flight}

The effect of forward flight on the aerodynamic forces generated by flexible wings is examined using anisotropic wings undergoing prescribed flap motion for a range of forward flight conditions. The results, shown in Figures 30 and 31 , are obtained for L1B1 and L3B1, $\beta_{0}=35^{\circ}, f=\{10,40\} \mathrm{Hz}$, and the forward flight conditions corresponding to the values given in Table 6 . The flapping motion is described by Eq. (31) and the magnitude of the free stream velocities were determined based on the tip speed of a rigid wing undergoing the same kinematics. The mean forces are obtained by time averaging the transient forces over cycles 2 through 5 . The mean forces computed for one wing were multiplied by a factor of two so as to approximate the force generated by a vehicle that has a pair of wings.

The mean lift and thrust generated by rigid and flexible wings are shown in Figure 30. The results indicate that the lift decreases (becomes more negative) with increase in forward flight speed; but the change in thrust is somewhat small. Also, different wings produce maximum mean thrust at different frequencies: L1B1 produces maximum thrust at $f=10 \mathrm{~Hz}$, whereas L3B1 produces the maximum thrust at $f=40 \mathrm{~Hz}$; similar trends were obtained for the case of hover.

The mean horizontal and vertical forces generated by the wings, indicative of the propulsive and payload capacity of the wings, are shown in Figure 31. The horizontal and vertical directions are shown in Figure 3 . Note that both $F_{h}$ and $F_{v}$ are positive in an actual vehicle; a negative value of $F_{h}$ denotes drag. The results show that $F_{v}$ increases and $F_{h}$ decreases with increase in forward flight speed, and wing flexibility has a beneficial influence. The flexible configurations have higher payload capacity and lower drag than rigid wings. Configurations L1B1 and L3B1 have the largest payload capacity and least drag at $10 \mathrm{~Hz}$ and $40 \mathrm{~Hz}$ respectively. These results indicate that the trends in force generation obtained for wings in hover also apply to forward flight.

Of the various wings and flapping frequencies considered in this study, positive values of both $F_{h}$ and $F_{v}$ were obtained only in the case of L3B1 at $f=40 \mathrm{~Hz}$, as shown in Figure 31(b). Thus, this case seems to be the most effective combination of wing flexibility and kinematics encountered in this study. The payload capacity for this case, which is 12 grams, is inadequate to support the expected weight of a potential vehicle (approximately 50 grams). This implies that further exploration of the parameter space is needed. Note that typical insect kinematics include a large pitching motion during and at the ends of each flapping stroke. In the current study the wing pitch was due to wing torsion. Therefore, the interaction of wing flexibility and kinematics that include active pitching of the wing has to be explored in greater detail. 


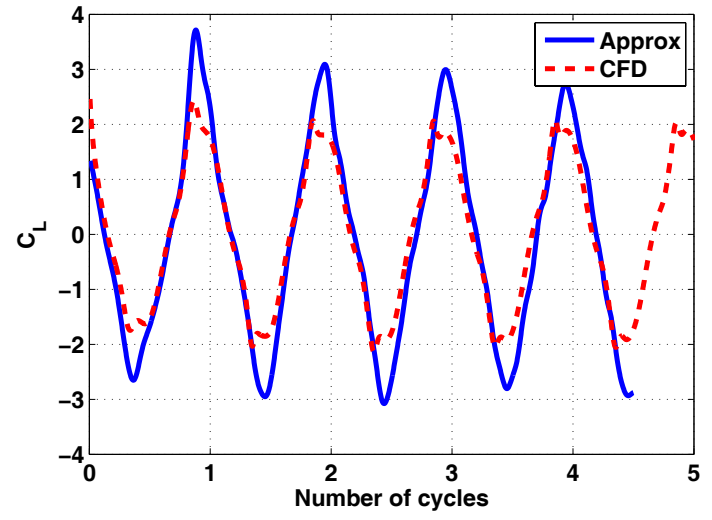

(a) $\mu=0.0$

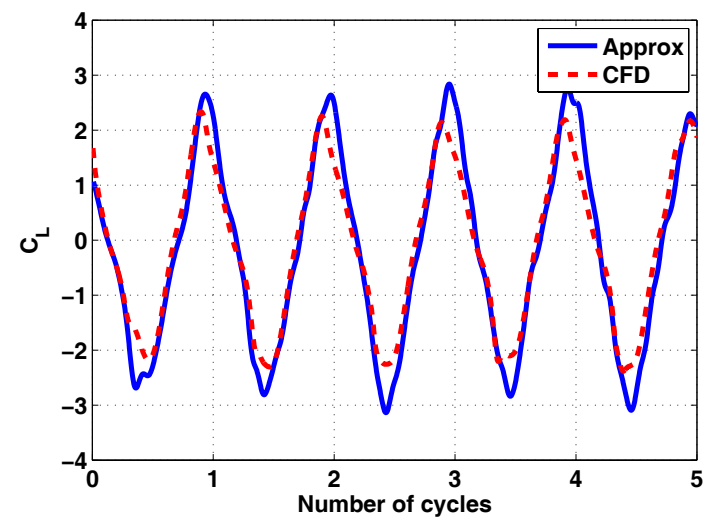

(c) $\mu=0.10$

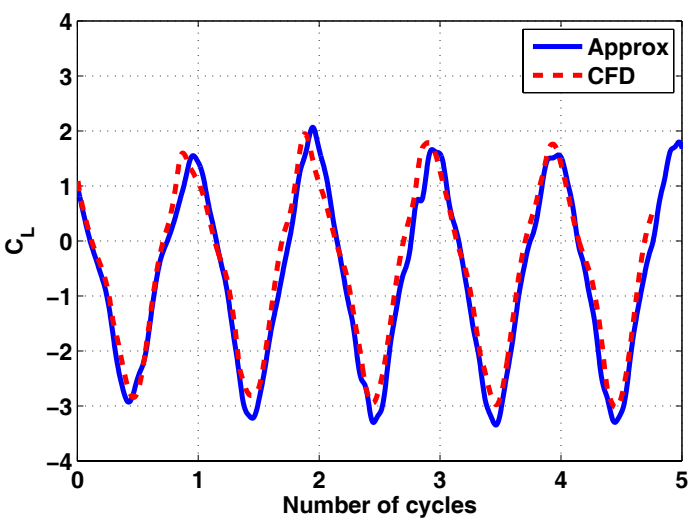

(e) $\mu=0.20$

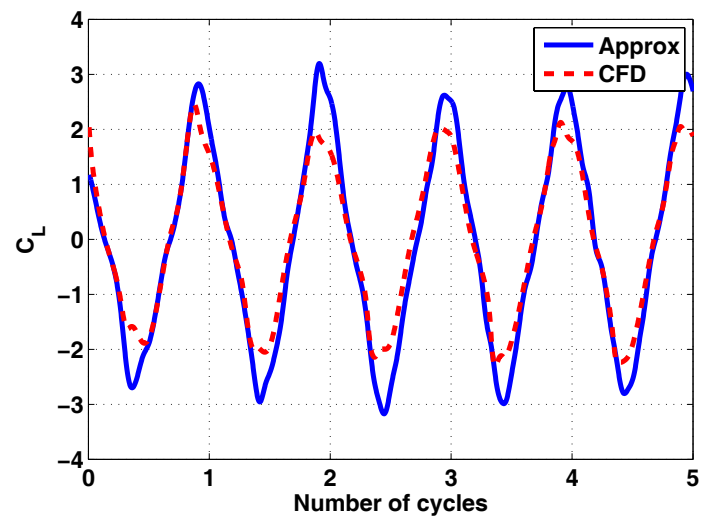

(b) $\mu=0.05$

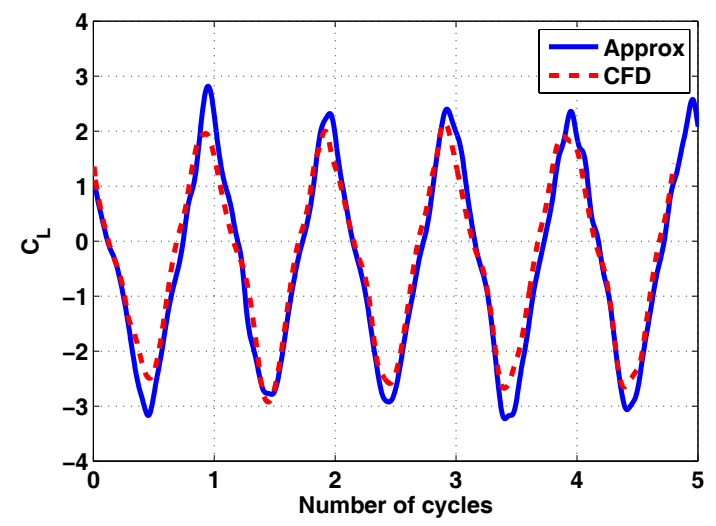

(d) $\mu=0.15$

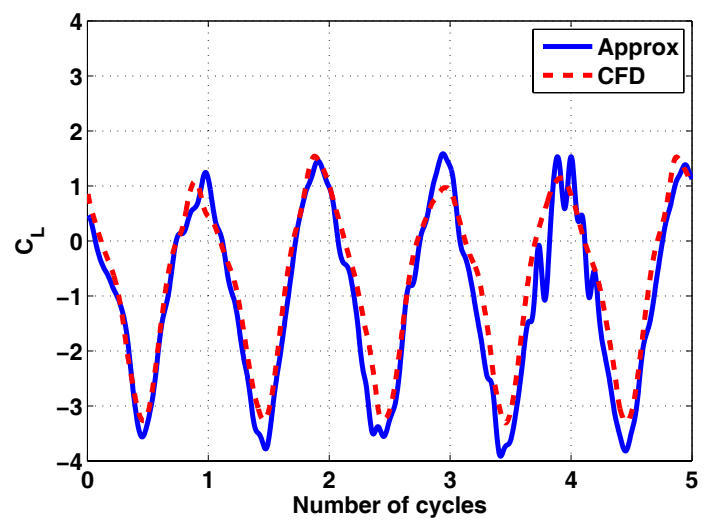

(f) $\mu=0.25$

Figure 20. Force coefficients generated by rigid wings in forward flight: $\beta_{0}=35^{\circ}, f=10 \mathrm{~Hz}$ 


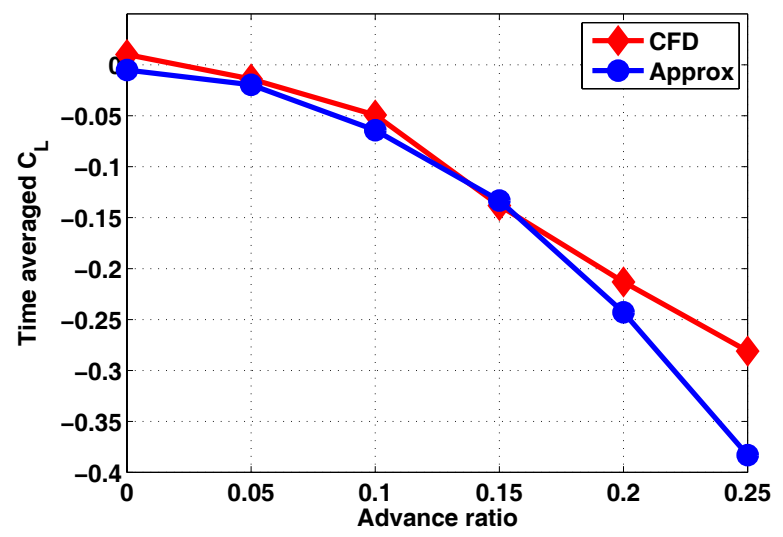

Figure 21. Mean lift generated by rigid wings in forward flight.

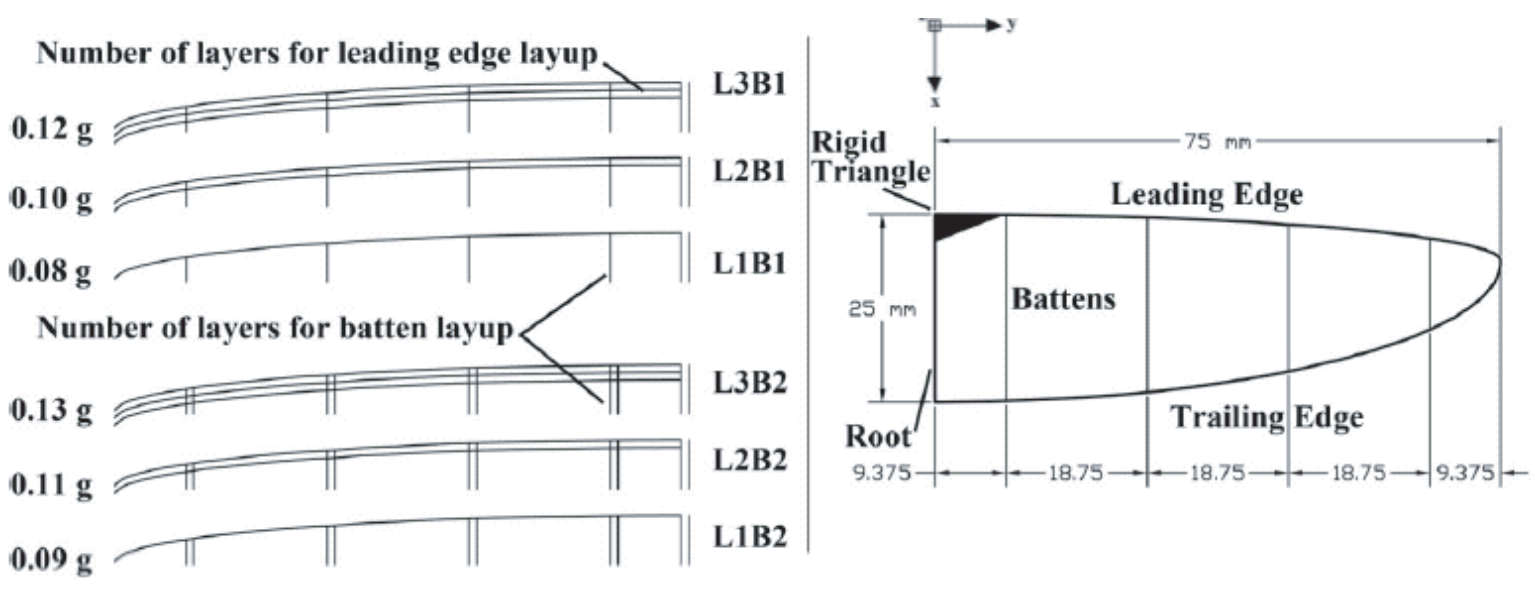

Figure 22. Anisotropic wing configurations, from Ref [33].

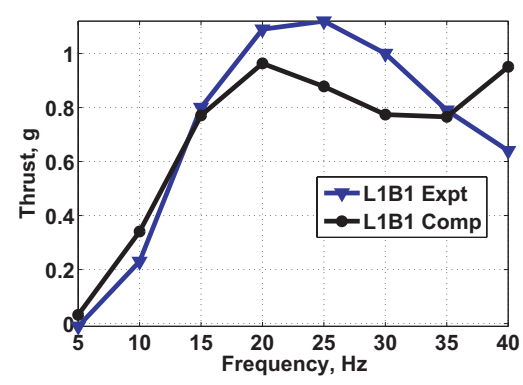

(a) L1B1

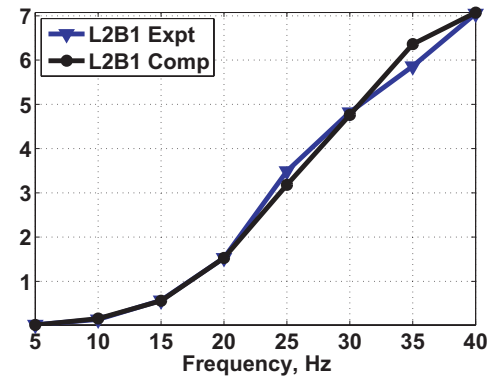

(b) L2B1

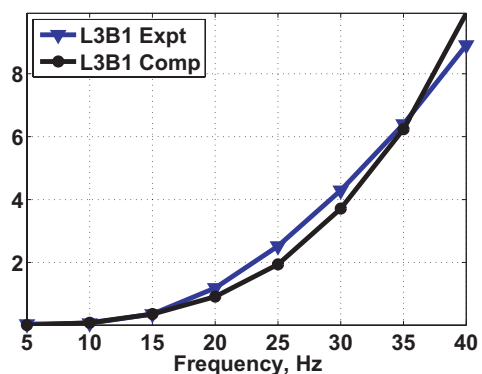

(c) L3B1

Figure 23. Comparison of thrust generated by anisotropic wings: 'Expt' - Experiments, ${ }^{33}$ 'Comp' - computations, current study 


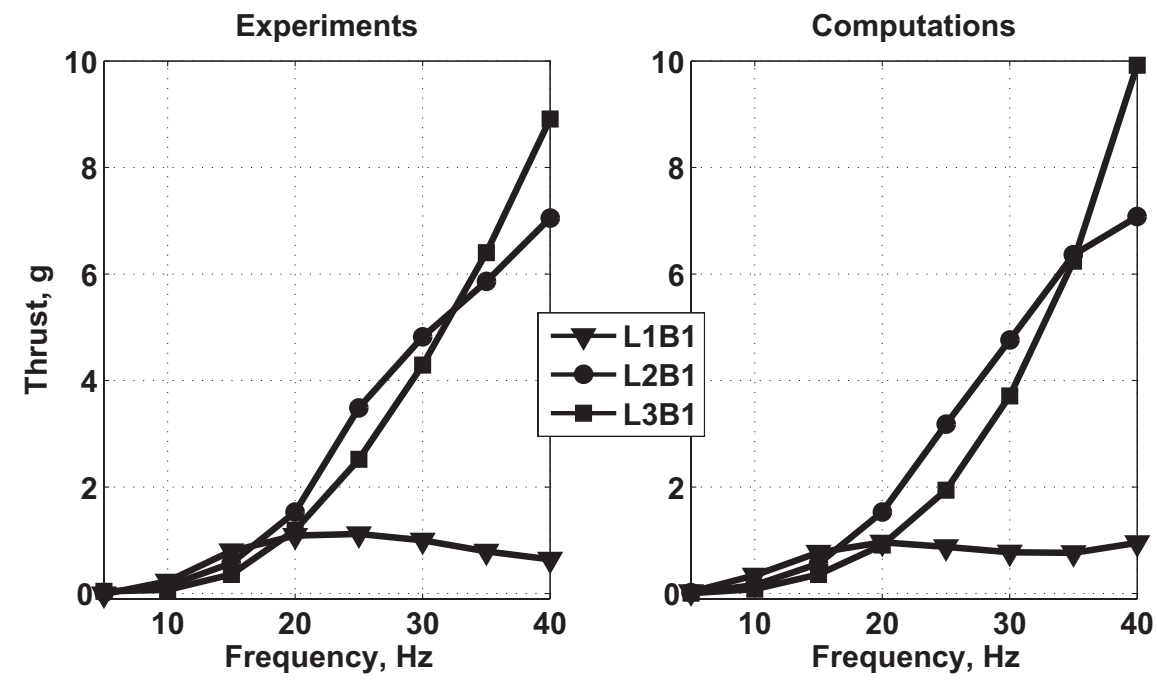

Figure 24. Thrust generated by one-layer batten configurations: Experiments, ${ }^{33}$ Computations - current study

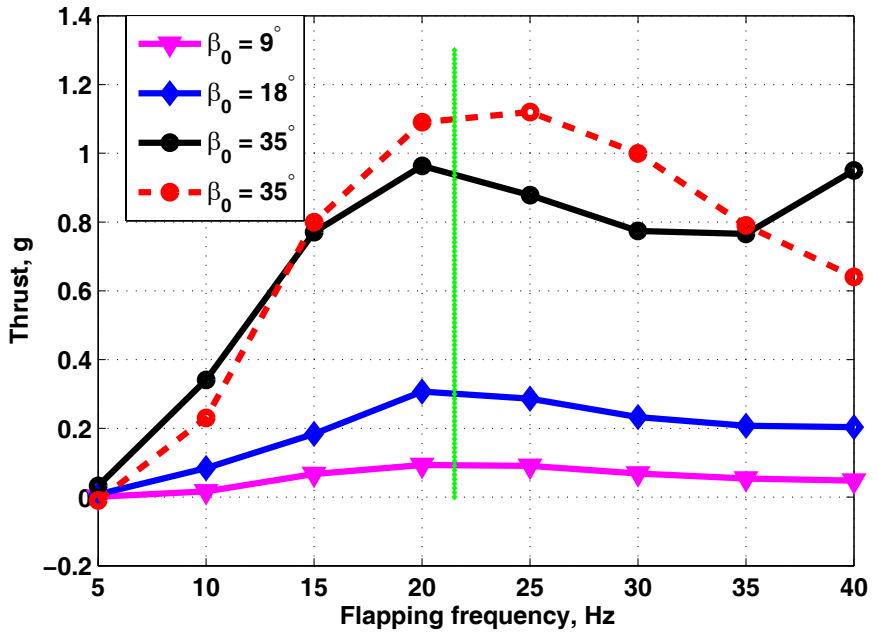

Figure 25. Thrust generated by L1B1 for various flapping amplitudes: Dashed line - Experiments, ${ }^{33}$ Solid lines - computations, current study. The vertical green line indicates the natural frequency of L1B1 

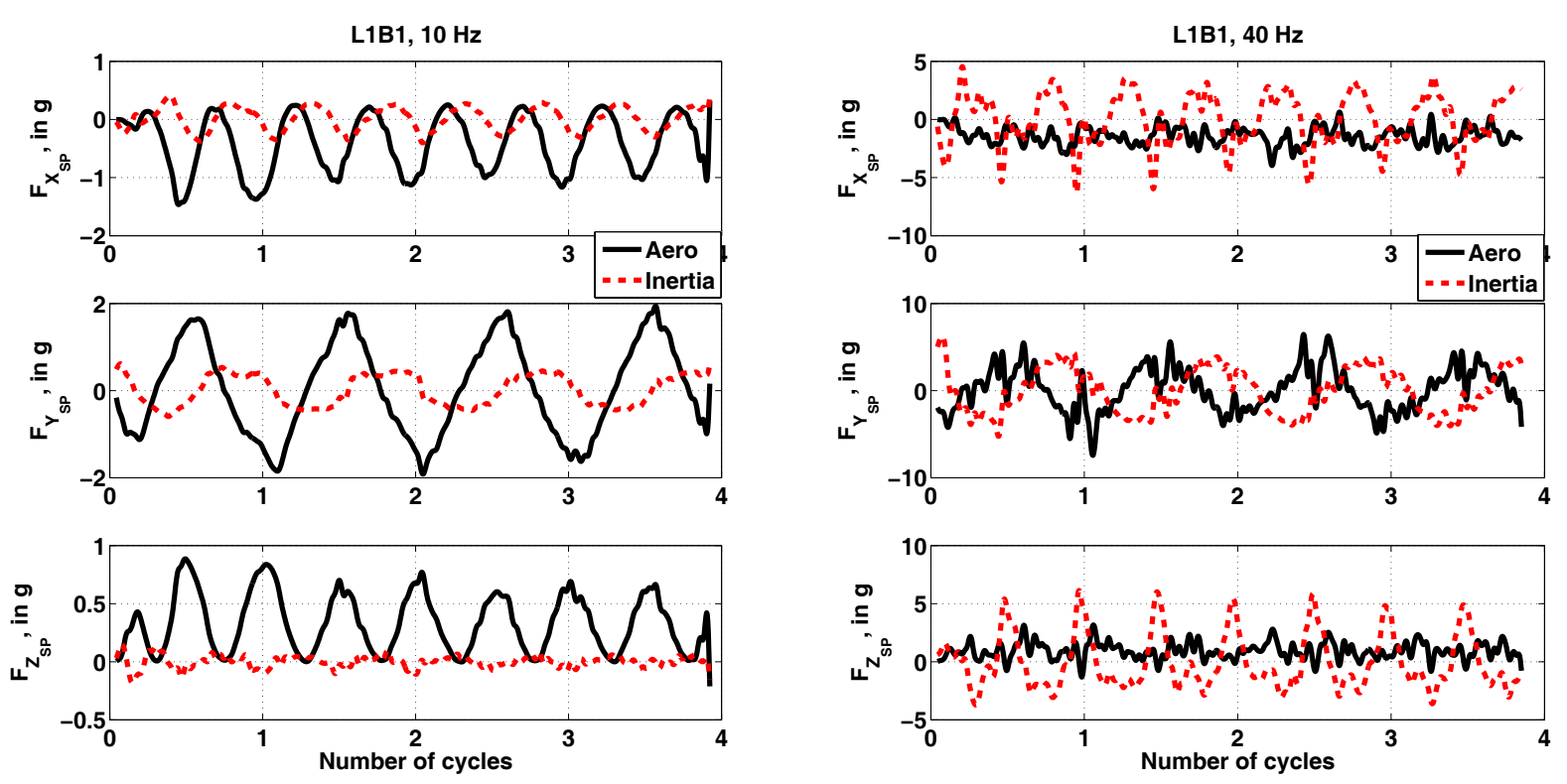
(a) $\mathrm{f}=10 \mathrm{~Hz}$
(b) $\mathrm{f}=40 \mathrm{~Hz}$

Figure 26. Aerodynamic and inertia loads acting on L1B1 in hover: $\beta_{0}=35^{\circ}$
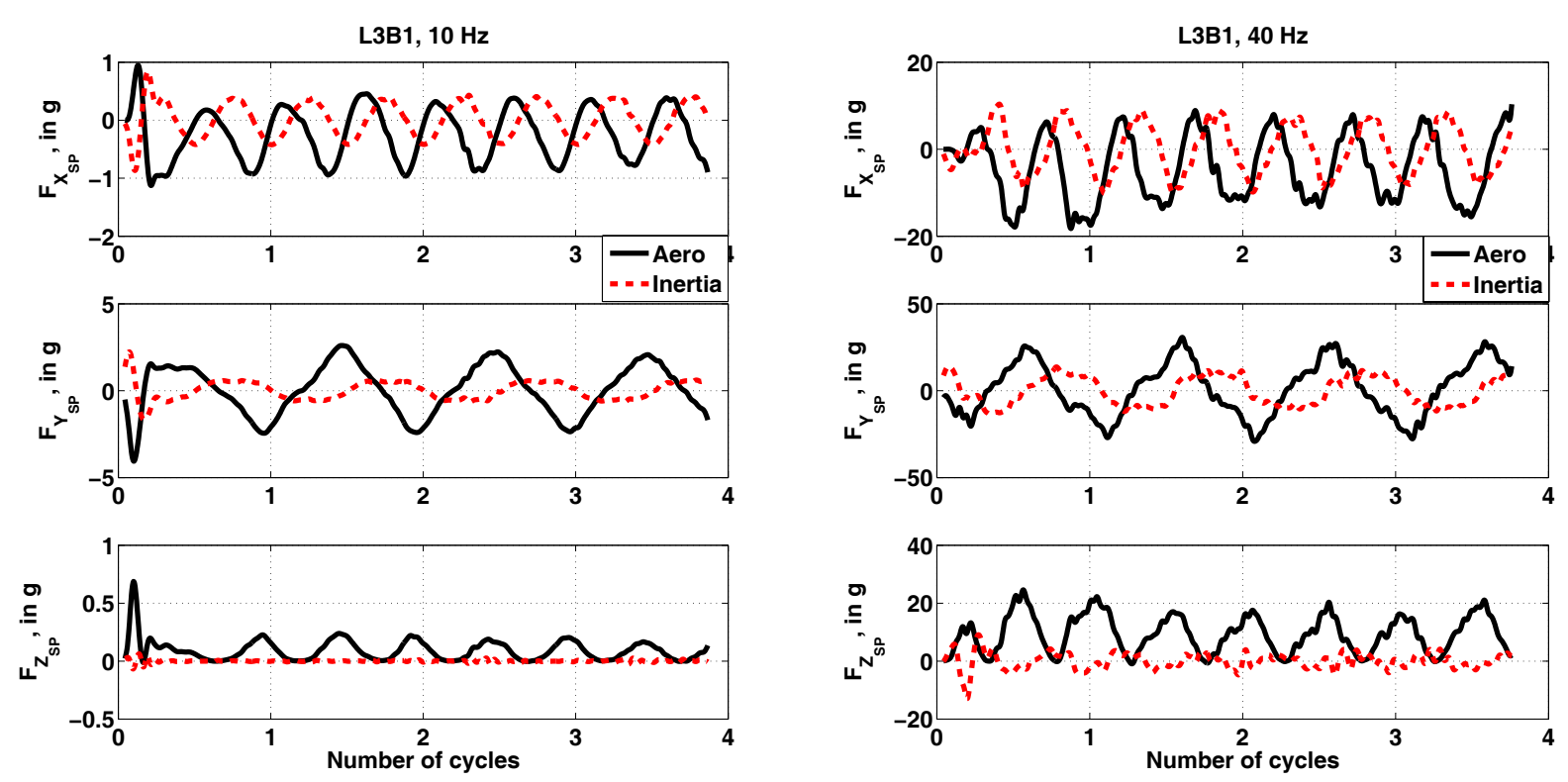
(a) $\mathrm{f}=10 \mathrm{~Hz}$
(b) $\mathrm{f}=40 \mathrm{~Hz}$

Figure 27. Aerodynamic and inertia loads acting on L3B1 in hover: $\beta_{0}=35^{\circ}$ 


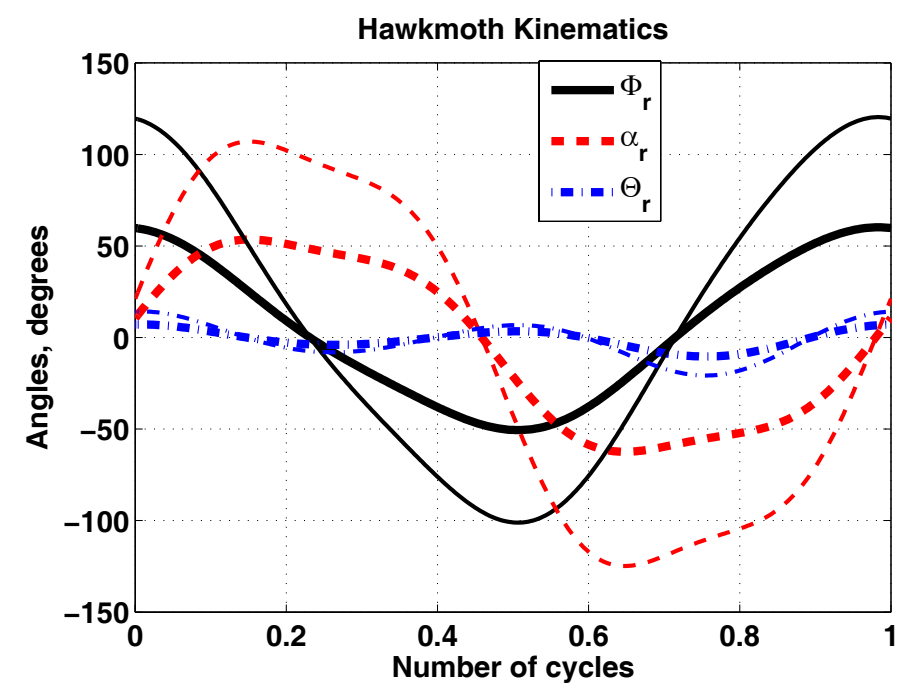

Figure 28. Hawkmoth kinematics: thin lines denote actual kinematics; ${ }^{27}$ thick lines denote scaled kinematics that are used in the current study.
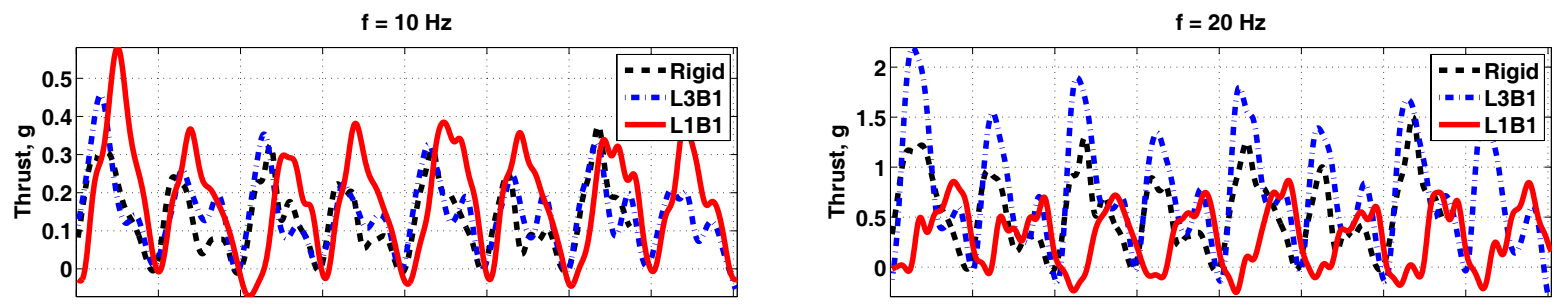

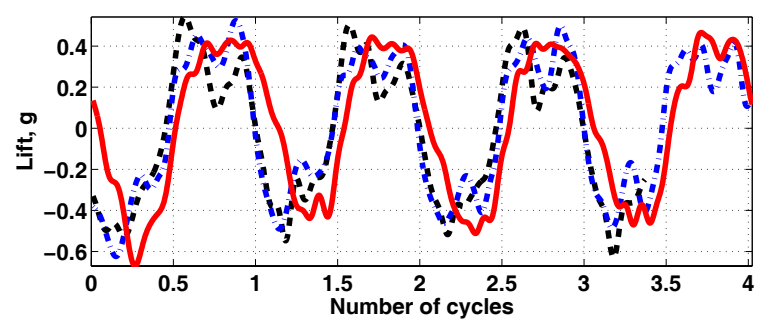

(a)

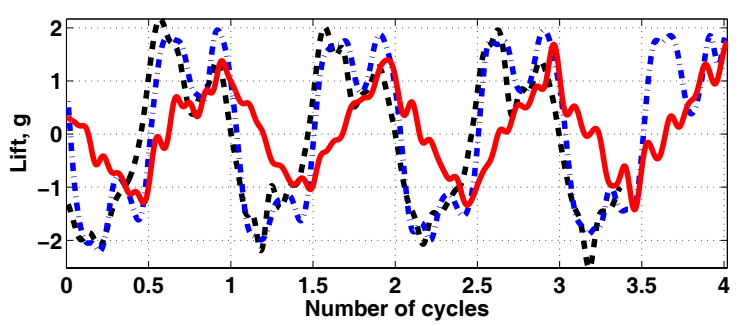

(b)

Figure 29. Lift and thrust generated by rigid and flexible wings undergoing scaled hawkmoth kinematics 


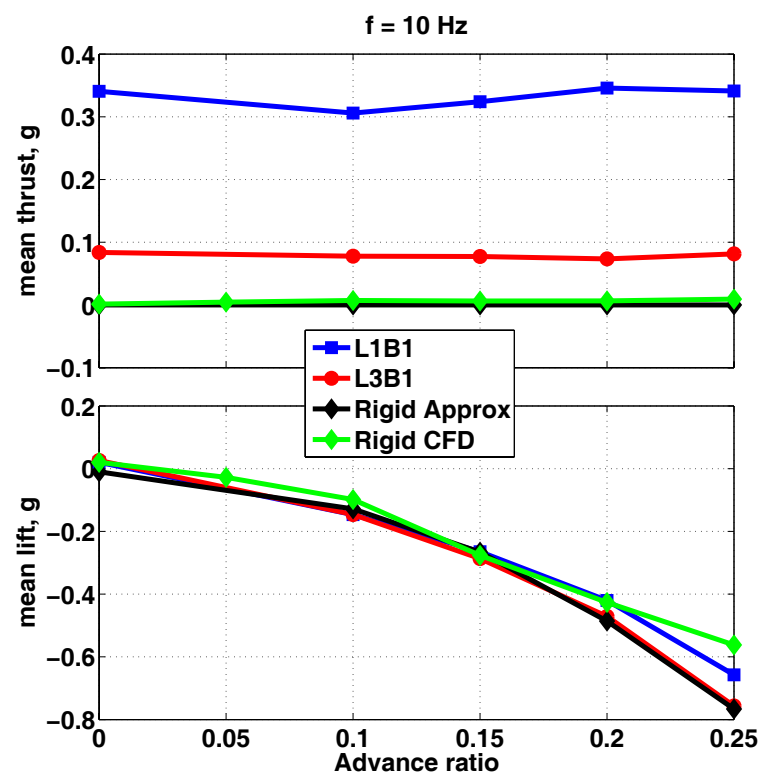

(a)

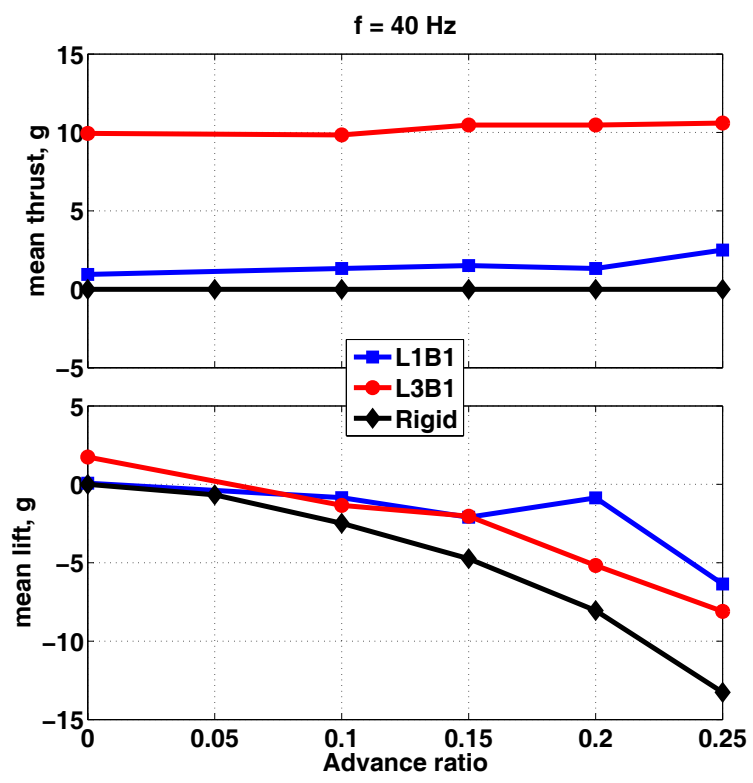

(b)

Figure 30. Mean lift and thrust, in grams, generated by rigid and flexible wings

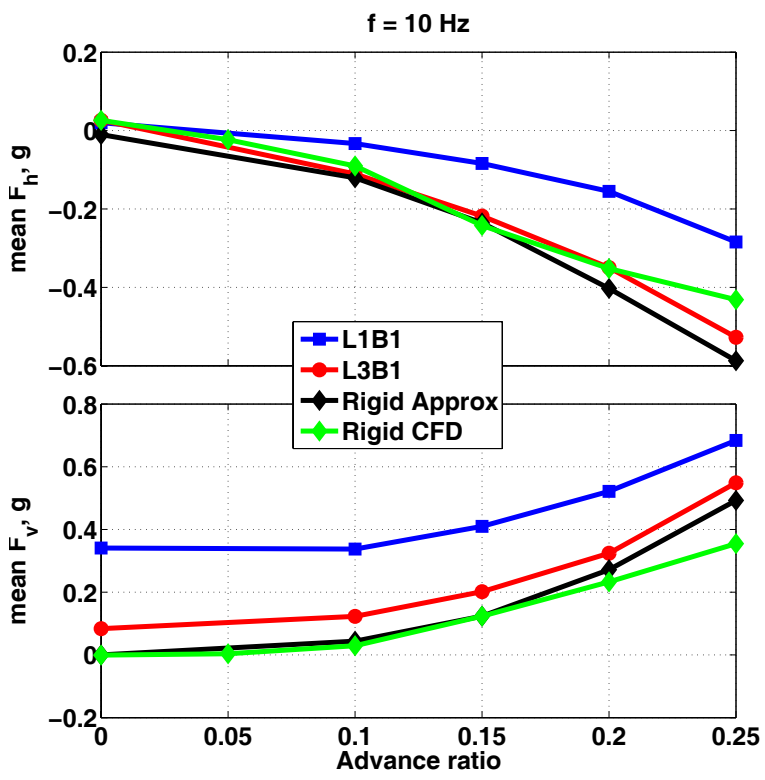

(a)

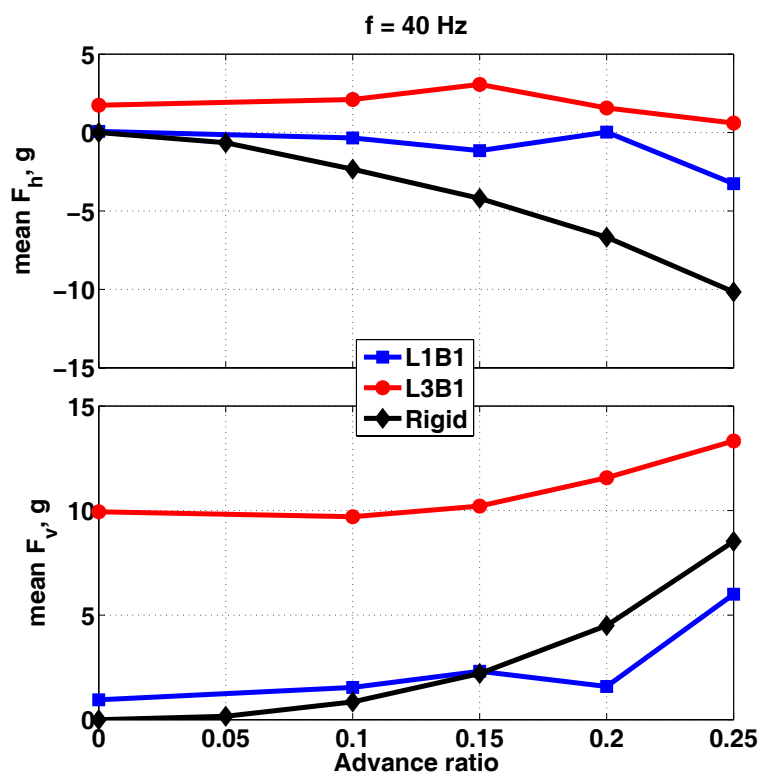

(b)

Figure 31. Mean horizontal and vertical forces, in grams, generated by rigid and flexible wings 


\section{Concluding Remarks}

The approximate aerodynamic model for flapping wings, originally developed for hover, has been extended to forward flight. The effect of viscosity was also incorporated in an approximate manner by including the temporal decay of shed vorticity into the calculation of induced velocity. The results were obtained for rigid airfoils, wings, and aeroelastic behavior of anisotropic wings.

1. Comparisons of forces generated by airfoils undergoing prescribed motions at $R e=100$ show that incorporating the effect of viscosity in the approximate model improved correlation with CFD based results.

2. The forces computed using the modified approximate aerodynamic model were compared with those obtained from CFD simulations for rigid Zimmerman wings in hover and forward flight. The approximate model shows reasonable agreement with CFD results and it captures the trends accurately.

3. Forces computed from the approximate aeroelastic model were compared with those obtained from experiments conducted on several Zimmerman wing configurations in hover. The approximate model shows reasonable agreement for the cases considered. In particular, a peak in thrust was obtained when the excitation frequency was close to the natural frequency of the wing, and the location of the peak is independent of the flapping amplitude.

4. Comparisons of inertia and aerodynamic loads acting on the wings indicate that the aerodynamic loads are comparable to inertia loads for the cases considered. This is contrary to what was found in previous studies. Therefore, it appears that the relative importance of aerodynamic and inertia loads in flapping wings is dependent on the configurations considered.

5. The payload capacity and propulsive capability of rigid and flexible wings in hover and forward flight were examined, and it was found that flexible wings have larger payload capacity and lower drag compared to rigid wings for the cases considered. Different flexible configurations perform better at different flapping frequencies. Thus, the choice of the 'best' flexible configuration depends on the flapping frequency. This behavior was also noted in hover.

6. Results for rigid and flexible wings undergoing insect-like kinematics suggest that the impact of wing flexibility on the force generation capacity of flapping wings may be dependent on the kinematics used.

\section{Acknowledgments}

This work was supported by the Air Force Office of Scientific Research's (AFOSR) MURI with Dr. Douglas R. Smith as Program Director. The first author would like to thank Patrick Trizila and Dr. Hikaru Aono for generating the CFD based results for the rigid airfoils and wings respectively.

\section{References}

\footnotetext{
${ }^{1}$ Mueller, T. J., Fixed and Flapping Wing Aerodynamics for Micro Air Vehicle Applications, Vol. 195, Progress in Aeronautics and Astronautics, published by AIAA, 2001.

${ }^{2}$ Platzer, M. E. and Jones, K., "Flapping Wing Aerodynamics - Progress and Challenges," $44^{\text {th }}$ AIAA Aerospace Sciences Meeting and Exhibit, Reno, Nevada, January 2006, AIAA Paper Number 2006-500.

${ }^{3}$ Sane, S. P., "The Aerodynamics of Insect Flight," The Journal of Experimental Biology, Vol. 206, 2003, pp. $4191-4208$.

${ }^{4}$ Ansari, S. A., Żbikowski, R., and Knowles, K., "Aerodynamic Modelling of Insect-like Flapping Flight for Micro Air Vehicles," Progress in Aerospace Sciences, Vol. 42, 2006, pp. 129 - 172.

${ }^{5}$ Shyy, W., Lian, Y., Tang, J., Liu, H., Trizila, P., Stanford, B., Bernal, L., Cesnik, C., Friedmann, P., and Ifju, P., "Computational Aerodynamics of Low Reynolds Number Plunging, Pitching, ad Flexible Wings for MAV Applications," $48^{\text {th }}$ AIAA Aerospace Sciences Meeting and Exhibit, Reno, Nevada, January 2008, AIAA Paper No. 2008-523.

${ }^{6}$ Shyy, W., Lian, Y., Tang, J., Viieru, D., and Liu, H., Aerodynamics of Low Reynolds Number Flyers, Cambridge University Press, 2008.

${ }^{7}$ Shyy, W., Aono, H., Chimakurthi, S., Trizila, P., Kang, C.-K., Cesnik, C., and Liu, H., "Recent Progress in Flapping Wing Aerodynamics and Aeroelasticity," Progress in Aerospace Sciences, Vol. 46, No. 7, 2010, pp. $284-327$.

${ }^{8}$ Baik, Y. S., Rausch, J. M., Bernal, L. P., Shyy, W., and Ol, M. V., "Experimental Study of Governing Parameters in Pitching and Plunging Airfoil at Low Reynolds Number," 48th AIAA Aerospace Sciences Meeting Including the New Horizons Forum and Aerospace Exposition, No. AIAA 2010-388, 4 - 7 January 2010, pp. 1-27.

${ }^{9}$ Bisplinghoff, R. L., Ashley, H., and Halfman, R. L., Aeroelasticity, Addison Wesley Co., 1955.
} 
${ }^{10}$ Singh, B. and Chopra, I., "Dynamics of Insect-Based Flapping Wings: Loads Validation," 47th AIAA/ASME/ASCE/AHS/ASC Structures, Structural Dynamics, and Materials Conference, No. AIAA 2006-1663, Newport, Rhode Island, 1-4 May 2006, AIAA Paper Number 2007-1757.

${ }^{11}$ Polhamus, E. C., "A Concept of the Vortex Lift of Sharp Edge Delta Wings Based on a Leading-Edge Suction Analogy," Tech. Rep. NASA TN D-3767, National Aeronautics and Space Administration, 1966.

12 Katz, J., "A Discrete Vortex Method for the Non-steady Separated Flow over an Airfoil," Journal of Fluid Mechanics, Vol. 102, 1981, pp. $315-328$.

${ }^{13}$ Shukla, R. K. and Eldredge, J. D., "An Inviscid Model for Vortex Shedding from a Deforming Body," Theoretical and Computational Fluid Dynamics, Vol. 21, 2007, pp. 343-368.

${ }^{14}$ Ansari, S. A., Żbikowski, R., and Knowles, K., "Non-linear Unsteady Aerodynamic Model for Insect-like Flapping Wing in the Hover. Part 1: Methodology and Analysis," Proceedings of the I MECH E Part G Journal of Aerospace Engineering, Vol. 220, No. 2, 2006, pp. 61-83.

${ }^{15}$ Ansari, S. A., Żbikowski, R., and Knowles, K., "Non-linear Unsteady Aerodynamic Model for Insect-like Flapping Wing in the Hover. Part 2: Implementation and Validation," Proceedings of the I MECH E Part G Journal of Aerospace Engineering, Vol. 220, No. 2, 2006, pp. 169-186.

${ }^{16}$ Gogulapati, A., Friedmann, P. P., Kheng, E., and Shyy, W., "Approximate Aeroelastic Analysis of Flapping Wings in Hover: Comparison with CFD and Experimental Data," 51st AIAA / ASME / ASCE / AHS / ASC Structures, Structural Dynamics, and Materials Conference, No. AIAA-2010-2707-967, Orlando, Florida, 12-15 April 2010.

${ }^{17}$ Combes, S. A. and Daniel, T. L., "Into thin air: contributions of aerodynamic and inertial-elastic forces to wing bending in the hawkmoth Manduca sexta," The Journal of Experimental Biology, Vol. 206, 2003, pp. 2999-3006.

${ }^{18}$ Daniel, T. L. and Combes, S. A., "Flexible Wings and Fins: Bending by Inertial or Fluid-Dynamic Forces," Integrative and Comparative Biology, Vol. 42, 2002, pp. $1044-1049$.

${ }^{19}$ Hamamoto, M., Ohta, Y., Hara, K., and Hisada, T., "Application of Fluid-Structure Interaction Analysis to Flapping Flight of Insects with Deformable Wings," Advanced Robotics, Vol. 21, No. 1-2, 2007, pp. 1-21.

${ }^{20}$ Gogulapati, A., Friedmann, P., and Shyy, W., "Nonlinear Aeroelastic Effects in Flapping Wing Micro Air Vehicles," 49th AIAA/ASME/ASCE/AHS/ASC Structures, Structural Dynamics, and Materials Conference, No. AIAA Paper Number 2008-1817, Schaumburg, IL, April 7 - 102008.

${ }^{21}$ Gogulapati, A., Friedmann, P., and Shyy, W., "Approximate Aeroelastic Analysis of Flapping Wings in Hover," International Forum for Aeroelasticity and Structural Dynamics, No. IFASD Paper Number 2009-143, Seattle, WA, June 22-25 2009.

${ }^{22}$ Chimakurthi, S. K., Tang, J., Palacios, R., Cesnik, C. E. S., and Shyy, W., "Computational Aeroelasticity Framework for Analyzing Flapping Wing Micro Air Vehicles,” 49th AIAA/ASME/ASCE/AHS/ASC Structures, Structural Dynamics, and Materials Conference, Schaumburg, Illinois, April 2008, AIAA Paper Number 2008-1814.

${ }^{23}$ Chimakurthi, S. K., Stanford, B. K., Cesnik, C. E. S., and Shyy, W., "Flapping Wing CFD/CSD Aeroelastic Formulation Based on a Co-rotational Shell Finite Element Formulation," 50th AIAA/ASME/ASCE/AHS /ASC Structures, Structural Dynamics, and Materials Conference, Palm Springs, California, May 2009, AIAA Paper Number 2009-2412.

${ }^{24}$ Aono, H., Chimakurthi, S., Wu, P., Sallstrom, E., Stanford, B., Cesnik, C., Ifju, P., Ukeiley, L., and Shyy, W., "A Computational and Experimental Study of Flexible Flapping Wing Aerodynamics," 48th AIAA Aerospace Sciences Meeting Including the New Horizons Forum and Aerospace Exposition, No. AIAA 2010-554, Orlando, Florida, 4-7 January 2010.

${ }^{25}$ Masoud, H. and Alexeev, A., "Resonance of flexible flapping wings at low Reynolds number," Physical review. E, Statistical, nonlinear, and soft matter physics, Vol. 81, No. 5, 2010, pp. 1-5.

${ }^{26}$ MSC. MARC, Volumes A - D, 2005.

${ }^{27}$ Willmott, A. P. and Ellington, C. P., "The Mechanics of Flight in the Hawkmoth Manduca Sexta: I. Kinematics of Hovering and Forward Flight," The Journal of Experimental Biology, Vol. 200, 1997, pp. 2705 - 2722.

${ }^{28}$ Saffman, P. G., Vortex Dynamics, Cambridge University Press, 1992.

${ }^{29}$ Gorelov, D. N., "Calculation of Pressure on an Airfoil Contour in an Unsteady Separated Flow," Journal of Applied Mechanics and Technical Physics, Vol. 49, No. 3, 2008, pp. 437-441.

${ }^{30}$ Bathe, K., Ramm, E., and Wilson, E. L., "Finite Element Formulations for Large Deformation Dynamic Analysis," International Journal for Numerical Methods in Engineering, Vol. 9, 1975, pp. 353 - 386.

${ }^{31}$ Bathe, K., Finite Element Procedures, Prentice-Hall Inc., 1996.

${ }^{32}$ Trizila, P., Kang, C.-K., Aono, H., Visbal, M., and Shyy, W., "Fluid Physics and Surrogate Modeling of a Low Reynolds Number Flapping Rigid Flat Plate," 28th AIAA Applied Aerodynamics Conference, No. AIAA 2010-5081, Chicago, Illinois, 28 June - 1 July 2010 , pp. $1-46$.

${ }^{33} \mathrm{Wu}$, P., Ifju, P., Stanford, B., Sallstrom, E., Ukeiley, L., Love, R., and Lind, R., "A Multidisciplinary Experimental Study of Flapping Wing Aeroelasticity in Thrust Production," 50th AIAA/ASME/ASCE/AHS/ASC Structures, Structural Dynamics, and Materials Conference, No. AIAA-2009-2413, Palm Springs, California, 4-7 May 2009.

${ }^{34}$ Aono, H., Kang, C., Cesnik, C. E. S., , and Shyy, W., "A Numerical Framework for Isotropic and Anisotropic Flexible Flapping Wing Aerodynamics and Aeroelasticity," 28th AIAA Applied Aerodynamics Conference, No. AIAA 2010-5082, Chicago, Illinois, 28 June - 1 July 2010, pp. 1-25.

${ }^{35}$ Kang, C.-K., Aono, H., Cesnik, C. S., and Shyy, W., "A Scaling Parameter for the Thrust Generation of Flapping Flexible Wings," 49th AIAA Aerospace Sciences Meeting Including the New Horizons Forum and Aerospace Exposition, No. AIAA 2011-1313, Orlando, Florida, 4-7 January 2011.

${ }^{36}$ Chung, J. and Hulbert, G. M., "A Family of Single-Step Houbolt Time Integration Algorithms for Structural Dynamics," Computational Methods in Applied Mechanics and Engineering, Vol. 118, 1994, pp. 1 - 11. 